\title{
A knowledge-based approach to multiple query processing
}

\author{
J.T. PARK ${ }^{*}$, T.J. TEOREY and S. LAFORTUNE \\ Computing Research Laboratory, Department of Electrical Engineering and Computer Science, University of \\ Michigan, Ann Arbor. MI 48109-2122, U.S.A.
}

\begin{abstract}
The collective processing of multiple queries in a database system has recently received renewed attention due to its capability of improving the overall performance of a database system and its applicability to the design of knowledge-based expert systems and extensible database systems. A new multiple query processing strategy is presented which utilizes semantic knowledge on data integrity and information on predicate conditions of the access paths (plans) of queries. The processing of multiple queries is accomplished by the utilization of subset relationships between intermediate results of query executions, which are inferred employing both semantic and logical information. Given a set of fixed order access plans, the $A^{*}$ algorithm is used to find the set of reformulated access plans which is optimal for a given collection of semantic knowledge.
\end{abstract}

Keywords. Database systems, Query optimization, Semantic knowledge.

\section{Introduction}

As knowledge-based systems are extended to more complex problems requiring large volumes of information and knowledge, the need for efficient processing of multiple queries and updates in a distributed environment becomes critical [7]. The handling of multiple queries is also found in extensions to existing database languages for the support of CAE applications such as VLSI design [9], and in deductive database systems [8].

An independent optimization of queries may overlook potential savings which can be achieved when queries are optimized collectively. We address the collective optimization of a set of queries such that, given a set of individual access plans of queries, a set of alternative access plans of queries which exhibits minimum cost is found using semantic knowledge on data objects.

The collective processing of batches of queries and update operations has been a popular technique in conventional file systems of the sixties and early seventies $[10,34]$. The majority of the research in this area has focused on the processing of multiple queries in centralized DBMSs $[6,8,10,14,16,28,32]$. We consider a new technique based on the concept of subquery relationship for the efficient processing of multiple transactions which occur almost simultaneously in both centralized and distributed computing environments. Both the knowledge on the semantic data integrity constraints and the information of logical predicate conditions of the access plans of queries are utilized in order to find a set of reformulated distributed query execution plans that exhibit minimum cost. The task of processing multiple queries is achieved by a rule-based expert system, Multiple Transaction Processor (MTP), which employs a planning technique combined with a problem solving method. The plan step

\footnotetext{
* Current address: Jong-Tae Park, Samsung Semiconductor \& Telecommunications Co., Ltd., San14, NongseoRi, Kihung-Eup Yongin-Kun, Kyungki-Do, Korea.
} 
infers the necessary constraints as in Dendral [18], and the problem solving step searches the state space to find an optimal solution using the $A^{*}$ algorithm [24].

Query processing can be categorized as individual or multiple. Individual query processing implies that each query is processed independently with respect to other queries $[13,39]$. Multiple query processing attempts to collectively optimize access plans of a set of queries occurring either simultaneously or not, by utilizing the commonality which exists among the set of queries in terms of accesses to relations, join/semi-join operations, and local physical data access $[5,6,8,10,14,16,26,28,32,34,36]$.

Multiple query processing may be further classified as semantic or nonsemantic. Semantic query processing implies that semantic knowledge such as functional dependencies and semantic data integrity constraints is utilized to achieve more efficient query processing $[4$, $11,12,18,15,25,35]$.

Finally, queries can be specified as either concurrent or nonconcurrent. In the concurrent case, a batch of transactions is assumed to occur almost simultaneously within a given time unit. The nonconcurrent case corresponds to the conventional data allocation problem in which frequencies of occurrences of transactions are given $[2,5,6,10,14,16,28,34,36,37]$. The processing of nonconcurrent multiple queries attempts to improve the overall system performance by storing or creating fast access paths via index or pointers for the intermediate results of queries which do not necessarily occur concurrently.

The assumptions that we make are as follows. A precompiled individual optimal access plan for each query is available. For example, the system $R^{*}$ query optimizer [21] generates the global plan for a query which is a procedural sequence of operations such as the accesses to relation, projection, join, inter-site transfers, and sorts whose estimated execution cost is minimal. The global plan is in a high-level form that lacks internal representations such as is parse tree structure or machine-executable code. Either joins or semi-joins or both are used as query processing tactic $[1,17,19]$. We assume that the speed of the computer network is relatively high such that the local processing cost cannot be negligible. The predicate conditions of a query are in a conjunctive form as assumed by other relevant research [39].

The paper is organized as follows. In Section 2, the subquery relationship is defined along with examples. In Section 3, the knowledge for efficient processing of multiple queries is described, and we present an algorithm for the reformulation of access plans of queries. In Section 4, the state space representation of the problem is formally presented, and the admissibility of the heuristic cost estimates for both general and simple cases is proved. The operation of MTP for a simple case is illustrated through an example in Section 5. Finally, a discussion on performance and some conclusions follow in Sections 6 and 7, respectively.

\section{View identification and subquery definition}

We assume that the reader is familar with the basic concepts of relational database theory [22]. Let $\operatorname{DOM}(A)$ be the domain of attribute $A$. Let $t, u, v$ and $w$ denote tuple variables. Consider relation $r$ on scheme $R$, and let $\Lambda$ denote an empty predicate formula. The standard relational operators will be denoted as follows: Projection, $\pi_{X}(r)$ with $X \subseteq R$; selection, $\sigma_{P}(r)$, with $P$ a 1st-order predicate formula; join (equi-join), $J_{P}\left(r_{1}, r_{2}\right)$, with $P$ a conjunction of equality clauses; and Cartesian product, $C\left(r_{1}, r_{2}\right)=J_{A}\left(r_{1}, r_{2}\right)$.

A relation in a database is referred to as a base relation. A view is the result of the execution of a relational operator such as selection $(\sigma)$, projection $(\pi)$, join $(J)$, Cartesian product $(C)$, union $(U)$ and difference $(D)$, on relations in a database. An access plan of a query is a sequence of relational operators applied to relations to get its result. When a view $V$ is a subset of another view $V^{\prime}$, we say that there is a subset relationship between two views $V$ and $V^{\prime}$. 
Processing multiple queries requires the identification of subset relationships between intermediate results (views) of queries, since some can be used for the processing of others. In a distributed environment, the recognition of these relationships among different access plans of queries can reduce the overall processing cost substantially by eliminating many expensive intersite joins. Such subset relationships can be inferred either from logical information of queries such as predicate conditions of queries, or from semantic information such as semantic data integrity constraints and functional dependencies existing among the attributes of each relation.

Example 2.1. Consider relations $r_{1}$ and $r_{2}$ with schemes $A B$ and $C D$ respectively, and two views $V_{1}=J_{P_{1}}\left(r_{1}, r_{2}\right)$ and $V_{2}=J_{P_{2}}\left(r_{1}, r_{2}\right)$, where $P_{1} \equiv(A=C)$ ard $P_{2} \equiv(A=C) \wedge(B=D)$. Since $P_{2}$ is more restrictive, we know that $V_{2}$ is a subset of or equal to $V_{1}$. In this case, the relationship between the join predicates is represented by $\forall u_{/ R_{1}} \forall v_{R_{2}}\left(P_{2}(u, v) \Rightarrow P_{1}(u, v)\right)$ in a closed well-formed formula. For notational convenience, it is denoted as $P_{2} \Rightarrow P_{1}$.

Example 2.1 illustrates that a subset relationship between views can be inferred from logical information on predicates of queries. We now illustrate that a subset relationship can also be inferred from the semantic knowledge on the database such as semantic data integrity constraints and functional dependencies.

Example 2.2. Consider an automobile insurance company which maintains a distributed database containing two relations OWNER and ISSUER at sites $S_{1}$ and $S_{2}$, respectively, and whose schemes are as follows: ISSUER (REPNAME, BRANCH) and OWNER (NAME, ADDRESS, AGE, SEX, INCOME, INSURANCE), where underlined attributes denote the primary key of the corresponding relation. REPNAME is the name of the insurance representative at the given branch of the company, and the other attributes are selfexplanatory.

We assume that the following semantic knowledge is obtained from the analysis of the user's requirements: "All representatives are living at cities at which they work, and, by managerial policy of the company, they are insured as owners at the branch where they work."

Consider two queries $Q T_{1}$ and $Q T_{2}$ which occur at site $S_{2}$; the first says "List the names of the owners insured by the company who work for the company as representatives"; and the second says "List the names of the owners insured by the company who live at cities where a branch of the company is located." From the knowledge, it is inferred that if the attribute NAME of OWNER is equal to REPNAME of ISSUER, then OWNER.ADDRESS is equal to ISSUER. BRANCH. Since the attributes NAME and REPNAME are keys of relations OWNER and ISSUER, respectively, it is found that $J_{P_{1}}\left(r_{1}, r_{2}\right)$ is a subset or equal to $J_{P_{2}}\left(r_{1}, r_{2}\right)$ where $r_{1}$ is OWNER; $r_{2}$ is ISSUER; $P_{1} \equiv($ NAME $=$ REPNAME) and $P_{2} \equiv($ ADDRESS $=$ BRANCH $)$. The relationship between the join predicates $P_{1}$ and $P_{2}$ associated with $Q T_{1}$ and $Q T_{2}$ is represented by the assertion, $\forall u_{/ R_{1}} \forall v_{/ R_{2}}$ $\left(P_{1}(u, v) \Rightarrow P_{2}(u, v)\right)$.

The access plan of a query can be represented by a query graph $\boldsymbol{G}$ [28] which is a triple $\left(N, E, f_{D}\right)$ where $N$ is a set cí nodes $E \subseteq N \times N$ is a set of directed edges, and $f_{D}: N \rightarrow 2^{2^{N}}$ is called the decomposition mapping for $\boldsymbol{G}$. Each node of $\boldsymbol{G}$ corresponds to a view, and it contains information on both the view and the processing method to obtain that view. $f_{D}\left(n_{i}\right)$ denotes the set of all possible sets of nodes in $N$ from which the view corresponding to $n_{i}$ can be constructed using suitable relational operations. The situation described in Example 2.2 is shown in Fig. 1a. It indicates that relation $r_{1}$ at site $S_{1}$ is transmitted to site $S_{2}$ to be joined 
with $r_{2}$ at site $S_{2}$, and the result of the join, either $V_{1}$ or $V_{2}$, is available at site $S_{2}$. If $\left(V_{1}, S_{2}\right)$ is replaced by $\left(V_{1}, S_{1}\right)$ in Fig. 1a, $r_{2}$ is transferred from site $S_{2}$ to site $S_{1}$ to be joined, and $V_{1}$ is available at site $S_{1}$. In this way, the distributed access plan of a query can be represented precisely.

Since $J_{P_{1}}\left(r_{1}, r_{2}\right) \subset J_{P_{2}}\left(r_{1}, r_{2}\right)$, view $V_{1}$ can be obtained by accessing view $V_{2}$ as shown in Fig. 1b. That is, two intersite joins (a) are replaced by one intersite join and one local selection (b). This can significantly reduce the total processing cost due to $I / O, \mathrm{CPU}$ and data transmission across the network. In this case, query $Q T_{1}$ is said to be a subquery of query $Q T_{2}$, meaning that the (intermediate) result of the former can be obtained from that of the latter. Alternatively, $Q T_{2}$ is called a superquery of $Q T_{1}$.

"View identification" addresses this recognition of subset relationships between access plans of two different queries to optimize collectively the processing of multiple queries. Generally, these relationships depend on both logical and semantic information, as shown in Examples 2.1 and 2.2. We will refer to such assertions as integrity constraints, denoted ICs. Throughout this paper, we assume that integrity constraints of the form $P_{1} \Rightarrow P_{2}$ are inferred from either logical or semantic information. (More details on view identification can be found in [25].)

It is assumed that a distributed query processing strategy processes all the (unary) projection operations before any binary relational operations, as in [3]. All the attributes of relations which are required for join conditions and target list of analyzed queries are assumed to be projected before the intermediate result is transferred to the next site.

\section{Multiple transaction processor: Reformulation algorithm}

Multiple Transaction Processor (MTP) is a rule-based expert system for the collective processing of multiple queries. Its operation is divided into two steps: a "plan step" and a "search step". The plan step infers integrity constraints which can be employed in the following search step for the generation of superqueries. The search step uses the $A^{*}$ heuristic search strategy [24]. In this paper, we shall only discuss the search step. The plan step is described in detail in [25]. It uses several heuristic rules to generate a set of ICs. This set is then pruned to retain only the "promising" ICs, where an IC is said to be promising if it can be used for the generation of a superquery.

We now describe the algorithm for the reformulation of access plans of queries during the search step. For simplicty, we omit site information in this section. The generalization to the case of a distributed database is straightforward (see next section). For the relational operations selection, projection and join, there are corresponding construction rules for reformulation.

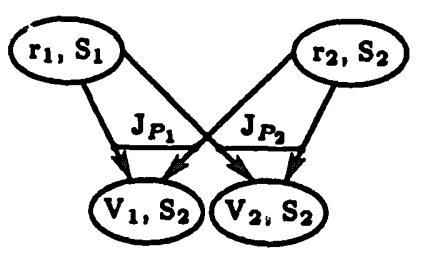

(a)

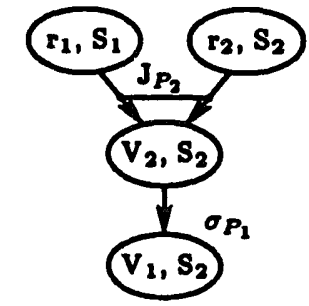

(b)

Fig. 1. Two joins are replaced by one join and one selection. 
Since join is considered to be the most expensive operation in both centralized DBMSs and distributed DBMSs, it is assumed that the access plan of a query is represented by a sequence of join operations where local unary relational operations are considered aggregately. Let $u o p_{i}$ be a unary relational operator. A sequence of unary operations $u_{o p_{i+k}}\left(u o p_{i+k-1}\left(\ldots\left(u_{o p}\left(V_{i}\right) \ldots\right)\right)\right)$ applied to a view $V_{i}$ is represented by $u_{o p}\left(V_{i}\right)$ for notational convenience. The access plan of a query is then represented by the sequence $\left\langle J_{P_{2}}\left(V_{1}, V_{2}\right), \quad J_{P_{4}}\left(V_{3}, V_{4}\right), \ldots, J_{P_{2 n}}\left(V_{2 n-1}, V_{2 n}\right)\right\rangle$ where, for $l=1,2, \ldots, V_{2 l}=u o p_{2 l}$ $\left(J_{P_{i}}\left(V_{j-1}, V_{j}\right)\right)$ and $V_{2 l-1}=u_{0 p_{2 l-1}}\left(J_{P_{i}}\left(V_{i-1}, V_{i}\right)\right)$ for some even $i, j, 2 \leqslant i, j<2 l$. Here, each join operation, $J_{P_{i}}\left(V_{i-1}, V_{i}\right)$ for $i=2,4, \ldots, 2 n$ in the access sequence, is defined as an access step of the sequence $\left\langle J_{P_{2}}\left(V_{1}, V_{2}\right), J_{P_{4}}\left(V_{3}, V_{4}\right), \ldots, J_{P_{2 n}}\left(V_{2 n-1}, V_{2 n}\right)\right\rangle$. In Theorem 1 of Section 4.2, each individual query access plan will be assumed to be optimal. But until then, this restriction is not enforced.

1. Construction rule for the selection operation.

Let us assume that there are two selection operations $\sigma_{Q_{1}}\left(V_{k}\right)$ and $\sigma_{Q_{2}}\left(V_{k}\right)$ where $V_{k}$ is a view, as shown in Fig. 2a. Suppose that there are two ICs, $Q_{1} \Rightarrow Q_{3}$ and $Q_{2} \Rightarrow Q_{3}$. The construction rule allows for the query graph on the left-hand side to be transformed into that on the right-hand side; two selection operations $\sigma_{Q_{1}}$ and $\sigma_{Q_{2}}$ are replaced by one selection operation $\sigma_{Q_{3}}$. It is noted that the views $V_{1}^{\prime}$ and $V_{2}^{\prime}$ can be derived from the view $V_{3}^{\prime}$ in the reformulated query graph as follows; $V_{1}^{\prime}=\sigma_{Q_{1}}\left(V_{3}^{\prime}\right), V_{2}^{\prime}=\sigma_{Q_{2}}\left(V_{3}^{\prime}\right)$.

2. Construction rule for the projection operation.

The projection operations $\pi_{W_{1}}\left(V_{3}^{\prime}\right)$ and $\pi_{w_{2}}\left(V_{3}^{\prime}\right)$ are replaced by the projection $\pi_{w_{1}} \cup w_{2}\left(V_{3}^{\prime}\right)$ as shown in Fig. 2b. The views $V_{1}^{\prime \prime}$ and $V_{2}^{\prime \prime}$ can be derived from the view $V_{3}^{\prime \prime}$ in the reformulated query graph since $V_{1}^{\prime \prime}=\pi_{w_{1}}\left(V_{3}^{\prime \prime}\right)$, and $V_{2}^{\prime \prime}=\pi_{W_{2}}\left(V_{3}^{\prime \prime}\right)$.

3. Construction rule for the join operation.

Let $V_{1}=J_{P_{1}}\left(V^{\prime}, V^{\prime \prime}\right)$ and $V_{2}=J_{P_{2}}\left(V^{\prime}, V^{\prime \prime}\right)$. If there are two ICs, $P_{1} \Rightarrow P_{3}$ and $P_{2} \Rightarrow P_{3}$, one join operation $J_{P_{3}}$ substitutes for two join operations $J_{P_{1}}$ and $J_{P_{2}}$. The views $V_{1}$ and $V_{2}$ can also be derived from the view $V_{3} ; V_{1}=\sigma_{P_{1}}\left(V_{3}\right)$ and $V_{2}=\sigma_{P_{2}}\left(V_{3}\right)$. Two join operations are replaced by one join operation, as shown in Fig. 3.

Now, let us look at a more general case in which the length of the query access plan is greater than one. For notational convenience, let us define $V_{1}, V_{2}, V_{4}$ and $V_{5}$ as follows: $V_{1}=J_{P_{1}}\left(\pi_{X_{1}}\left(V_{i}\right), \pi_{X_{2}}\left(V_{j}\right)\right), V_{2}=J_{P_{2}}\left(\pi_{X_{1}}\left(V_{i}\right), \pi_{X_{2}}\left(V_{j}\right)\right), V_{4}=J_{P_{4}}\left(\pi_{Y_{1}}\left(V_{1}\right), \pi_{W_{1}}\left(\sigma_{Q_{1}}\left(V_{k}\right)\right)\right)$, and $V_{5}=J_{P 5}\left(\pi_{W_{2}}\left(\sigma_{Q_{2}}\left(V_{k}\right)\right)\right)$. Suppose that there are two queries whose access plans are described below.
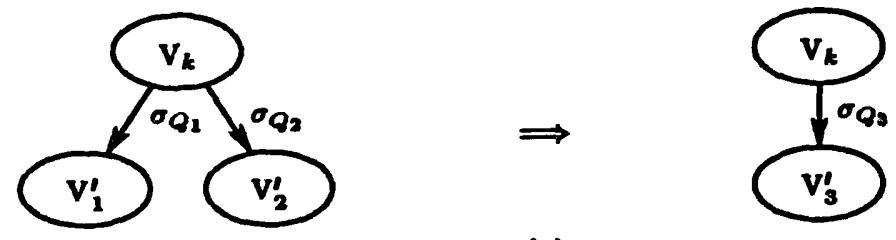

(a)
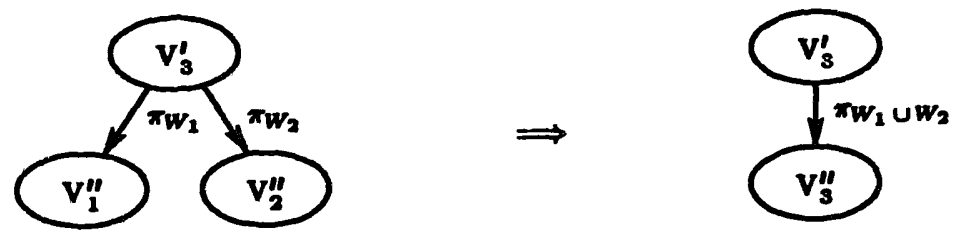

(b)

Fig. 2. Construction rules for (a) selection and (b) projection operations. 


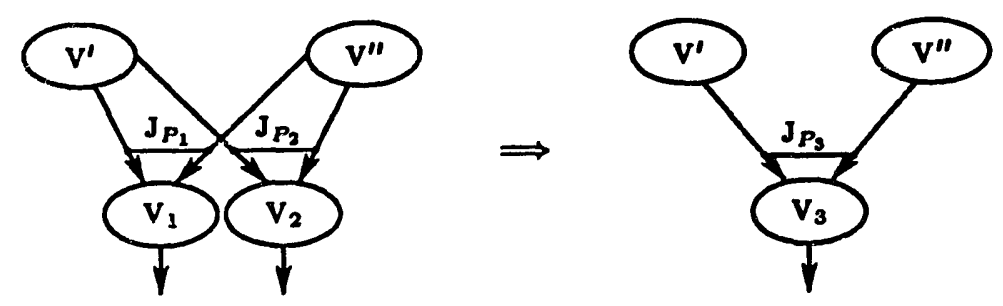

Fig. 3. Construction rule for the simple join operation.

- access-plan $\left(Q T_{l}^{r_{t}}\right):\left\langle J_{P_{1}}\left(\pi_{X_{1}}\left(V_{i}\right), \pi_{X_{2}}\left(V_{j}\right)\right), J_{P_{4}}\left(\pi_{Y_{1}}\left(V_{1}\right), \pi_{W_{1}}\left(\sigma_{Q_{1}}\left(V_{k}\right)\right)\right), \pi_{Z_{1}}\left(V_{4}\right) \cdots\right\rangle$

- access-plan $\left(Q T_{m}^{r_{m}}\right):\left\langle J_{P_{2}}^{1}\left(\pi_{X_{1}}\left(V_{i}\right), \pi_{X_{2}}\left(V_{j}\right)\right), J_{P_{5}}\left(\pi_{Y_{2}}\left(V_{2}\right), \pi_{W_{2}}\left(\sigma_{Q_{2}}\left(V_{k}\right)\right)\right), \pi_{Z_{2}}\left(V_{5}\right) \cdots\right\rangle$

(The superscripts $r_{l}$ and $r_{m}$ denote the set of relations referenced by queries $Q T_{l}$ and $Q T_{m}$, respectively.)

The following ICs are assumed to be inferred from the knowledge base; $P_{1} \Rightarrow P_{3}, P_{2} \Rightarrow P_{3}$, $Q_{1} \Rightarrow Q_{3}, Q_{2} \Rightarrow Q_{3}, P_{4} \Rightarrow P_{6}$, and $P_{5} \Rightarrow P_{6}$. The rule for the simple join operation in Fig. 3 , using ICs $P_{1} \Rightarrow P_{3}$ and $P_{2} \Rightarrow P_{3}$, allows to obtain the reformulated query graph shown in Fig. 4a. Here, it is said that a superquery $Q G_{l}^{\left\{V_{i}, V_{j}\right\}}$ of $Q T_{l}^{r_{l}}$ and $Q T_{m}^{r_{m}}$ is generated whose access plan is $J_{P_{3}}\left(\pi_{X_{1}}\left(V_{i}\right), \pi_{X_{j}}\left(V_{j}\right)\right)$.

The current access step, denoted CAS, of a query (superquery) is defined to be the most recently reformulated access step of the access plan of the query. The current view of a query is defined as the result of the execution of the current access step of the query. The CAS of both $Q T_{l}^{r_{l}}$ and $Q T_{m}^{r_{m}}$ is $J_{P_{3}}\left(\pi_{X_{1}}\left(V_{i}\right), \pi_{X_{2}}\left(V_{j}\right)\right)$ in Fig. 4a. Since the number of eliminated intersite join operations tends to be proportional to the length of the access sequence of the superquery, it may be a good strategy for the reduction of the total processing cost to stretch out the superquery $Q G_{1}^{\left\{V_{i}, V_{j}\right\}}$ to $Q G_{1}^{\left\{V_{i}, V_{j}, V_{k}\right\}}$ such that the intermediate results $V_{4}$ and $V_{5}$ of the executions of the queries $Q T_{l}^{r_{l}}$ and $Q T_{m}^{r_{m}}$ can be obtained from $Q G_{1}^{\left\{V_{i}, V_{i}, V_{k}\right\}}$. Exploiting the knowledge $Q_{1} \Rightarrow Q_{3}, Q_{2} \Rightarrow Q_{3}, P_{4} \Rightarrow P_{6}$, and $P_{5} \Rightarrow P_{6}$ in the search step, the query graph of Fig. $4 \mathrm{a}$ is transformed into that of $4 \mathrm{~b}$. It is noted that $V_{4}$ and $V_{5}$ can be obtained from $V_{6}$ which is the result of the execution of $Q G_{1}^{\left\{V_{i}, V_{j}, V_{k}\right\}}$. In this case, $Q G_{1}^{\left\{V_{i}, V_{j}\right\}}$ is said to be extended into $Q G_{1}^{\left\{V_{i}, V_{j}, V_{k}\right\}}$. Here, two join operations are again replaced by one join operation. This motivates the following heuristic in the search step:

Generate a superquery whose access plan is as long as possible.

In order to describe the above construction rule in more detail, we need to introduce some definitions. Let $V=\sigma_{P}\left(r_{i}\right)$. $\operatorname{attr}(P)$ is defined as the set of all attributes appearing in the selection predicate $P$. For example, if $V=\sigma_{(A=B) \wedge\left(C={ }^{\prime} \text { value" }\right)}\left(r_{i}\right), \operatorname{attr}((A=B) \wedge(C=$ "value")) $=A B C$. Similarly, $\operatorname{lattr}(P)$ and $\operatorname{rattr}(P)$ are defined for the join predicate $P$. Let $V=J_{P}\left(r_{i}, r_{j}\right)$ with the schemes of $r_{i}$ and $r_{j}$ being $R_{i}$ and $R_{j}$ respectively. lattr $(P)$ is defined as the set of all attributes appearing in both $P$ and $R_{i} ; \operatorname{rattr}(P)$ as the set of all attributes in both $P$ and $R_{j}$. For example, $\operatorname{lattr}(P)=A$ and $\operatorname{rattr}(P)=B$ for $J_{R_{i}, A=R_{j} \cdot B}\left(r_{i}, r_{j}\right)$.

We now describe the construction rule of the join operation in Fig. 4. First, the conditions for the extensibility of superquery $Q G_{1}^{\left\{V_{i}, V_{j}\right\}}$ are checked. This consists of the identification of any ICs with respect to join predicates $P_{4}$ and $P_{5}$. If this condition is satisfied, we then check the feasibility of extending the superquery by verifying the conditions related to the local selection and projection operations. Since these conditions are satisfied by $P_{4} \Rightarrow P_{6}$ and $P_{5} \Rightarrow P_{6}$, and $Q_{1} \Rightarrow Q_{3}$ and $Q_{2} \Rightarrow Q_{3}$, we try to construct the intermediate result from which both intermediate results of $Q T_{l}^{r_{l}}$ and $Q T_{m}^{r_{m}}$ can be obtained. This construction of the intermediate result of the superquery requires the projection operation $\pi_{R_{1}}$ to be carried out, in order to provide the necessary attributes and the corresponding data for the selection 


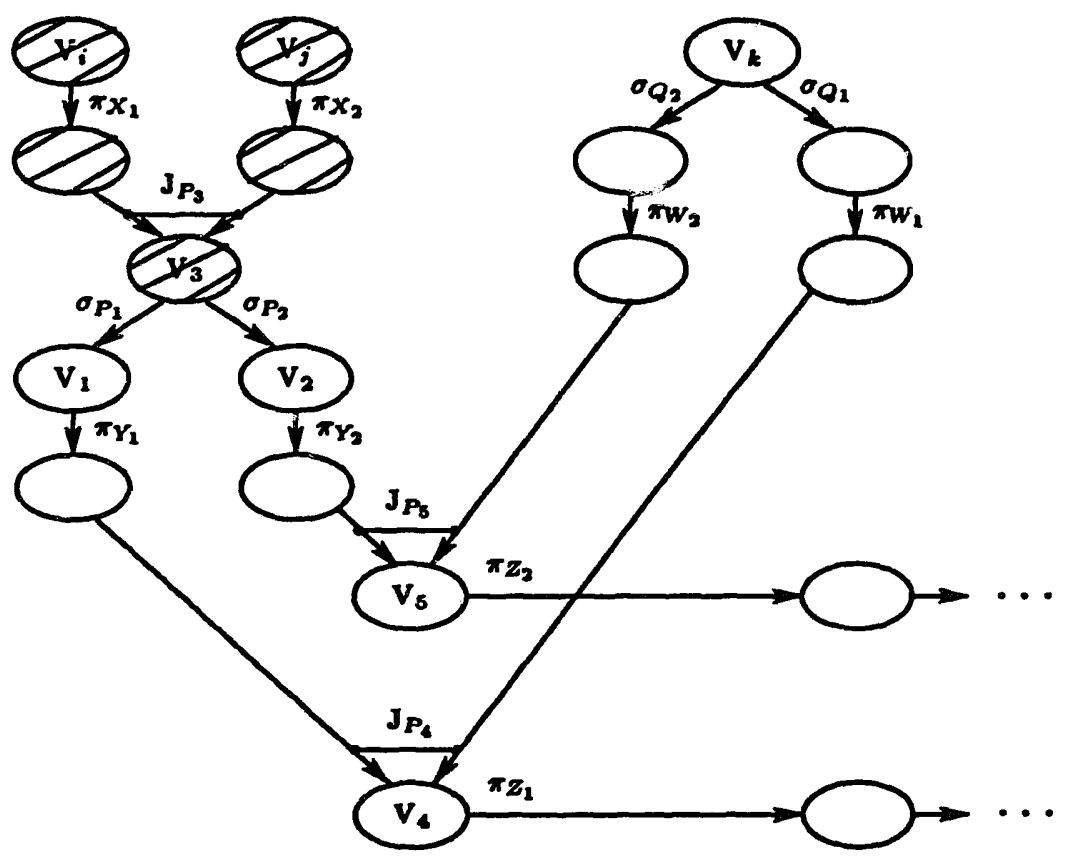

(a)

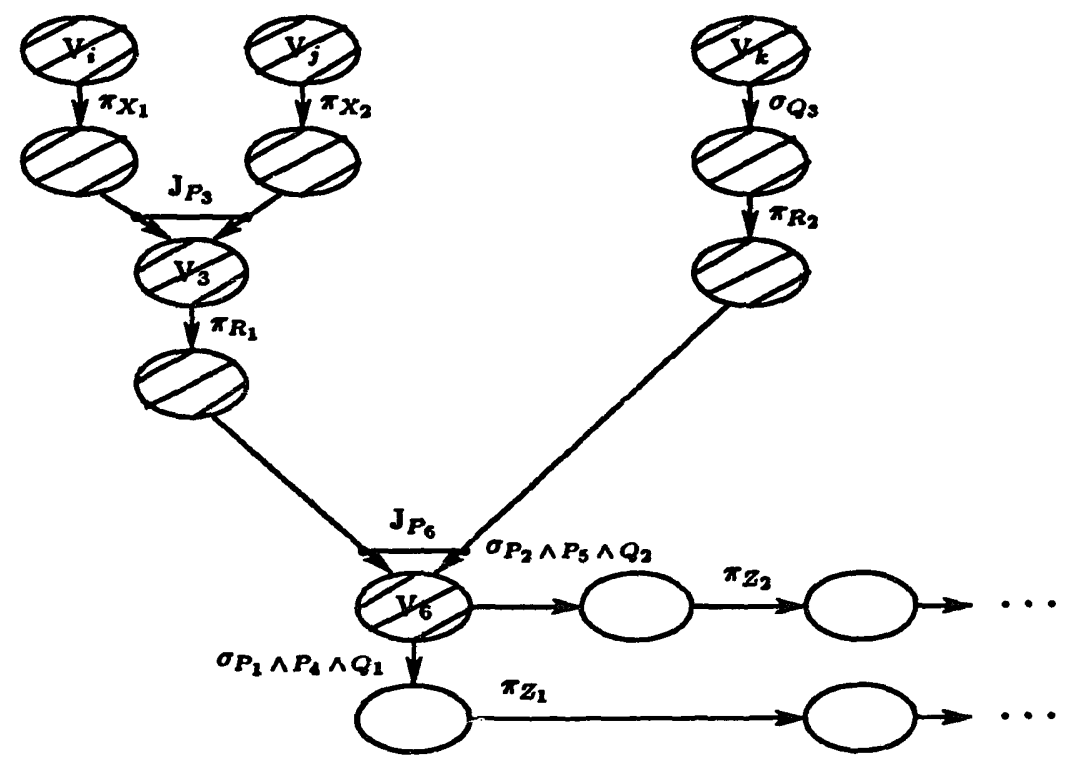

(b)

Fig. 4. Construction rule including unary and binary operations.

operations $\sigma_{P_{1}}$ and $\sigma_{P_{2}}$ to be executed at the next access step. It must also provide the necessary attributes for the join operation $J_{P_{6}}$. Here, $R_{1}=\left(\cup_{i=1}^{2} Y_{i}\right) \cup\left(\cup_{i=1}^{2} \operatorname{attr}\left(P_{i}\right)\right) \cup$ lattr $\left(P_{6}\right)$.

Second, the construction rules for the local selection and projection operations, $\sigma_{Q_{1}}$ and $\sigma_{Q_{2}}$, are applied to the view $V_{k}$. The join operation $J_{P_{6}}$ also requires the attribute $\operatorname{lattr}\left(P_{6}\right)$ to be projected. The selection operation $\sigma_{Q_{1}}$ and $\sigma_{Q_{2}}$ are also deferred to the next stage, which requires the attributes $\operatorname{attr}\left(Q_{1}\right)$ and $\operatorname{attr}\left(Q_{2}\right)$. Since all these projection operations can be 
performed simultaneously in a centralized DBMS, all the attributes involved in the projection operations can be combined. This composite attribute is represented by $R_{2}$ with $R_{2}=W_{1} \cup W_{2} \cup \operatorname{rattr}\left(P_{6}\right) \cup \operatorname{attr}\left(Q_{1}\right) \cup \operatorname{attr}\left(Q_{2}\right)$.

Finally, the local selection operations $\sigma_{P_{1}}$ and $\sigma_{P_{2}}$, and $\sigma_{Q_{1}}$ and $\sigma_{Q_{2}}$, are postponed to the next access step where these are carried out together with the selection operations $\sigma_{P_{4}}$ and $\sigma_{P_{5}}$, respectively, to get $V_{4}$ and $V_{5}$ from $V_{6}$.

When the above transformation occurs, it is said that a state transition occurs from the current state represented by Fig. $4 \mathrm{a}$ to the state corresponding to Fig. 4b. This state transition is defined as a one-step transition since only one intersite join operation is accounted for the state transition. All the local selection and projection operations involved in an intersite join operation are considered to be executed at the access step associated with that join operation.' The CAS is also changed to $J_{P_{6}}\left(\pi_{R_{1}}\left(V_{3}\right), \pi_{R_{2}}\left(\sigma_{Q_{3}}\left(V_{k}\right)\right)\right)$ for both queries. In the above one-step transition, one join, one selection, and two projection operations are saved. If $Q_{3}=Q_{2}, \sigma_{P_{2} \wedge P_{5} \wedge Q_{2}}$ is equivalent to $\sigma_{P_{2} \wedge P_{5}}$. Furthermore, if $P_{5} \equiv P_{6}$, then $\sigma_{P_{2} \wedge P_{5}} \equiv$ $\sigma_{P_{2}}$. All the views which are involved in the generation of a superquery up to the current access step in Fig. 4 are marked by $/ / /$.

The above construction rules for the general case are summarized below.

Step 1. Check the feasibility of extending a superquery. There must be promising join and selection conditions for the predicate formulas in the access plans of the subqueries.

Step 2. If it is feasible, then compose $R_{1}$ and $R_{2}$.

Step 3. Apply the construction rules for the local selection and projection operations related to $V_{k}$.

Step 4. Extend the access plan of the superquery by replacing two join operations of a subquery by one join operation, and postpone all the join, selection and projection operations as shown in Fig. $4 a$ and $b$.

Step 5. Update CAS and mark the views which are involved in the reformulated access plans.

During the plan step, we can also identify the equivalence relationship $P_{4} \Leftrightarrow P_{5}$. In this case, we can further reduce the search space as follows. Let us assume that the attributes appearing in $\pi_{R 1}$ and $\pi_{R_{2}}$ are $R_{4}$ when $P_{6}$ is replaced with $P_{4}$, and $R_{5}$ when $P_{6}$ is replaced with $P_{5}$. We further assume that $\operatorname{SIZE}\left(R_{4}\right) \geqslant \operatorname{SIZE}\left(R_{5}\right)$. The heuristic is as follows.

If $P_{4} \Leftrightarrow P_{5}$, then select $P_{5}$ as the predicate for the superquery, i.e. let $P_{5}$ play the role of $\boldsymbol{P}_{6}$ in Fig. 4. Since $\operatorname{SIZE}\left(R_{4}\right) \geqslant \operatorname{SIZE}\left(R_{5}\right), \operatorname{VOL}\left(V_{6}\right)$ derived using $J_{P_{4}}$ is always greater than or equal to that derived using $J_{P_{5}}$. This guarantees that we always select the smaller volume of intermediate results in the process of superquery generation associated with $P_{4}$ and $P_{5}$. Since we do not generate the superquery associated with IC $P_{5} \Rightarrow P_{4}$, the subtree using $P_{5} \Rightarrow P_{4}$ can be pruned off in the search space.

\section{Multiple transaction processor: Search step}

\subsection{Formal representation of problem space}

In this section, we present formal definitions for state, initial and goal states, heuristic cost evaluation functions, and we describe the rules in the search step. These consist of generation and test rules which together embody the $A^{*}$ search strategy.

\footnotetext{
'This aggregation of local unary operations and an intersite join operation is considered for the efficient representation of a state transition.
} 
Let $Q T_{-} C O S T_{k}$ be the processing cost for the execution of the query $Q T_{k}$ along its access plan in a distributed environment. Let $G$ be a query graph which is constructed by integrating all the individual access plans of queries. The objective is to reformulate the access plans in $G$ using the available knowledge such that the total processing cost over $n$ queries, $\Sigma_{k=1}^{n} Q T_{-} C O S T_{k}$, is minimized.

A state is informally defined as a set of access plans which are represented by a query graph with attached proper information required to estimate the total processing cost in a distributed environment. A state transition occurs whenever a new query graph is constructed by adding or modifying relational operations, thereby reformulating the access plans of the queries, using both logical (syntactic) and semantic knowledge under the constraint that all the views in the current state can be obtained from the new state. This state transition occurs by the activation of the generation rules described below. The generation rules allow for two types of state transitions to occur; one is advancing an access step of a query, and the other merges two current access steps of two different queries and generates a superquery according to the construction rule of the previous section. Both types of state transitions are subject to the following constraints: (i) any view at the current state should be derivable from the newly generated one; (ii) the state transition is only possible using the knowledge associated with the current access step (one-step transition).

A state $\omega$ is formally defined as a 5-tuple $(G, A P, C A S, g(\omega), \hat{h}(\omega))$. $G$ is a query graph which is constructed by integrating all the individual access plans (see e.g. Figs 10-13). $A P$ is the set of (reformulated) access plans of queries inferred from $G$ :

$$
\boldsymbol{A P}=\left\{\operatorname{access}-p \operatorname{lan}\left(Q T_{k}\right) \mid k=1,2, \ldots, n\right\} .
$$

When a query is using the (intermediate) results of another query or superquery, $Q G_{k}$, its access plan should be reformulated as follows: $\left\langle\right.$ access-plan $\left.\left(Q G_{k}\right), V_{i}, V_{i+1}, \ldots, V_{m}\right\rangle . C A S$ is the set of all the current access steps of queries:

$$
C A S=\left\{C A S \text { of } Q T_{k} \mid k=1,2, \ldots, n\right\} .
$$

The value $g(\omega)$ is the sum of all the processing costs of queries from their initial access steps to their respective current access steps along their (reformulated) access sequence. The value $\hat{h}(\omega)$ is the estimate of the total remaining cost for all the queries from the current state to reach the goal state, using the strategy of multiple transaction processing.

\subsection{Generation rules}

We describe the cost functions $g$ and $\hat{h}$, and the generation and test rules in more detail. There are three generation rules: $I$-rule, $M$-rule, and $F$-rule. $I$-rule moves forward the access step of the query along the access sequence to reflect the individual distributed query processing strategy. $M$-rule tries to generate a profitable superquery by merging access plans of queries, and $F$-rule is useful for the cost evaluation at the end of the superquery generation steps. The firing of the generation rules is controlled by the specificity ordering of the conflict resolution strategy. $M$-rule has more priority than $I$-rule and $F$-rule, and $I$-rule has more priority than $F$-rule. These priorities reflect one of the inference-guiding heuristics in the search step.

Let $\left\langle V_{1}, V_{2}, \ldots, V_{n}\right\rangle$ be the access sequence of query $Q T_{k}$. I-rule is described as follows:

I-rule: Move forward one-step further the current access step of the query along the access sequence $\left\langle V_{1}, V_{2}, \ldots, V_{n}\right\rangle$. Initially, the $C A S$ of $Q T_{k}$ is set to $V_{1}$. The activation of $I$-rule enables the $C A S$ of $Q T_{k}$ to become $V_{2}$; the next firing to become $V_{3}$ and so on. 
When there are two current access steps for which promising integrity constraints exist, $M$-rule reformulates the access plans of the queries to generate a superquery relationship by invoking the construction rule described in Section 3.

M-rule: If there exist promising ICs which can be applied to views in the current access steps of two different queries, then reformulate the access plans using the construction rule, and make the newly generated access step to be the current access steps of the corresponding queries.

The answer to a query can be represented by $\boldsymbol{u o p}_{n}\left(V_{n}\right)$ for some "final" view $V_{n}$ of the query. Since it cannot move forward any further, we introduce the final view $V_{f}$ such that $V_{n}$ can be advanced to it without incurring any processing cost. This is actually a stopping rule.

F-rule: If the result of the current access step of the query $Q T_{k}$ is view $V_{n}$ where the answer to the query is equal to $u_{0} p_{n}\left(V_{n}\right)$, then move forward the current access step to $V_{f}$.

$I$-rule, $M$-rule and $F$-rule are complete in the sense that all the possible ways of reformulating the access plans of queries can be enumerated along the access sequences within a given knowledge. They are also nonredundant since each rule is fired only once using given ICs. Finally, the search step is informed by using the priority existing between $I$-rule, $M$-rule and $F$-rule as well as being guided by the heuristic cost evaluation function $\hat{h}$ which will be described subsequently. Note that the identification of the same views can be facilitated by using ICs of the form $P_{i} \Rightarrow P_{i}$.

For an access step $J_{P}\left(u o p_{i}\left(V_{i}\right), u_{0} p_{j}\left(V_{j}\right)\right)$, we want to allow for various access strategies which take into account the different intersite communication costs and different local processing costs. In order to do that, we describe the cost function associated with a query graph where each node of the query graph contains site information. Let $V_{k}=J_{P_{k}}\left(u o p_{i}\left(V_{i}\right)\right.$, $\left.\operatorname{uop}_{j}\left(V_{j}\right)\right)$. The access step $J_{P_{k}}\left(\operatorname{uop}_{i}\left(V_{i}\right)\right.$, uop $\left.\left(V_{j}\right)\right)$ is represented by the query graph in Fig. 5.

Here, since the oval contains site information, we represent the node of the query graph by the notation $V S_{k}$ where $V S_{k}$ is defined to be an ordered pair $\left(V_{k}, S_{k}\right)$, denoting view $V_{k}$ located at site $S_{k}$.

We describe the processing cost evaluation functions. $\psi_{s_{l}}^{l}(u o p, V)$ is defined as the local processing cost to carry out the relational operator uop to view $V$ at site $S_{i} ; \psi_{s_{i} s_{j}}^{c}(V)$ as the communication cost to transmit the volume of view $V, V O L(V)$, from site $S_{i}$ to site $S_{j}$, and $\psi_{s_{i}}^{\prime}\left(J_{P_{k}}, V, V^{\prime}\right)$ as the local processing cost to carry out the join operation $J_{P_{k}}$ for the views $V$ and $V^{\prime}$ at site $S_{i}{ }^{2}$ For a unary operation $\operatorname{uop}\left(V_{i}\right)$, the corresponding query graph is shown in Fig. 6, where $V_{j}=u o p\left(V_{i}\right)$.

If $S_{i}=S_{j}$ in Fig. 6, uop is performed at site $S_{i}$. Otherwise, uop is performed at site $S_{i}$, but the result $V_{j}$ of its execution is transmitted to site $S_{j}$, and stored there for further processing.

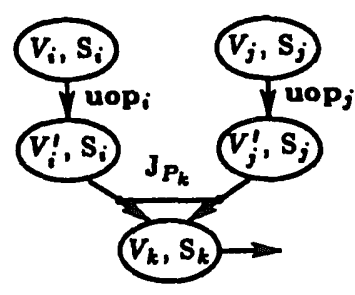

Fig. 5. The access step of $J_{p_{k}}\left(\operatorname{uop}_{i}\left(V_{1}\right)\right.$, uop, $\left.\left(V_{,}\right)\right)$.

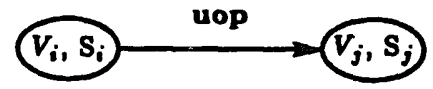

Fig. 6. The access step of $u o p\left(V_{i}\right)$.

\footnotetext{
2 If necessary, other cost functions can be defined; for example, semi-join operations can be considered.
} 
The corresponding cost function, $\operatorname{cst}\left(V S, V S_{j}\right)$ with $V S=\left\{V S_{i}\right\}$ is defined as follows.

$$
\operatorname{cst}\left(V S, V S_{j}\right)= \begin{cases}\psi_{s_{i}}^{\prime}\left(\text { uop }, V_{i}\right) & \text { for } S_{i}=S_{i} \\ \psi_{s_{i}}^{\prime}\left(u o p, V_{i}\right)+\psi_{s_{i} s_{j}}^{c}\left(V_{j}\right) & \text { for } S_{i} \neq S_{j} .\end{cases}
$$

Going back to the query graph in Fig. 5, we assume that all the unary operations are carried out before any intersite data transmission occurs. However, the join operation $\delta_{p}$ can be performed at different sites according to the following protocol. If $S_{k}=S_{i}$, it is assumed that view $V_{j}^{\prime}$ at site $S_{j}$ is transmitted to site $S_{i}$, and $J_{p}$ is carried out on $V_{i}^{\prime}$ and $V_{j}^{\prime}$ at site $S_{i}$ where the result $V_{k}$ is stored. If $S_{k} \neq S_{i}$ and $S_{k} \neq S_{j}$, both $V_{i}^{\prime}$ and $V_{j}^{\prime}$ are transmitted to site $S_{k}$, and the join is performed at site $S_{k}$. The result $V_{k}$ is also stored at site $S_{k}$. According to the above protocol, every distributed query access plan using the joins as processing tactic can be represented precisely by the above modified query graph. We describe the cost formula for each case below. Let $V S=\left\{V S_{i}, V S_{j}\right\}$ where $V S_{i}=\left(V_{i}, S_{i}\right)$ and $V S_{j}=\left(V_{j}, S_{j}\right)$. Let $V S_{k}=\left(V_{k}, S_{k}\right)$. The corresponding cost formula, $\operatorname{cst}\left(V S, V S_{k}\right)$, is shown below.

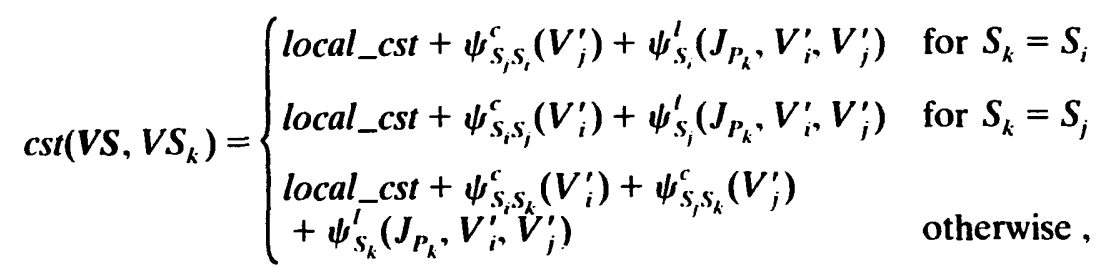

where local_cst $=\psi_{s_{i}}^{\prime}\left(u_{o p}, V_{i}\right)+\psi_{s_{t}}^{l}\left(u o p_{j}, V_{j}\right)$ and where $V_{i}^{\prime}=u o p_{i}\left(V_{i}\right), V_{j}^{\prime}=u o p_{j}\left(V_{j}\right)$. Note that we do not assume any specific cost model for the evaluation of the access step.

Let $V S_{k}$ be a view which is an intermediate result from the execution of a query along its access plan. Let $V S$ be the set of views such that $V S \in V S$ implies that $V S$ is involved in the part of the access plan yielding $V S_{k}$. VS_COST $\left(V S_{k}\right)$ determines the processing cost to get $V S_{k}$ along the access plan of the query, and it is defined as follows:

$$
V S_{-} \operatorname{COST}\left(V S_{k}\right)=\operatorname{cst}\left(V S, V S_{k}\right)+\sum_{v S \in V S} V S_{-} \operatorname{COST}(V S) \text {. }
$$

Assume that state $\omega$ consists of $m$ superqueries, $Q G_{l}$ for $l=1,2, \ldots, m$, and $n$ original queries $^{3} Q T_{k}^{r_{k}}$ for $k=1,2, \ldots, n$ where the current view of the query $Q T_{k}^{r_{k}}$ is denoted as $V S_{k}=\left(V_{k}, S_{k}\right)$. Let $V S_{l}^{\prime}$ be the result of executing $Q G_{l}$, and $N Q G_{l}$ be the number of queries $Q T_{k}^{r_{k}}$ which use the intermediate result $V S_{l}^{\prime}$ to get their current view $V S_{k}$. Then,

$$
g(\omega)=\sum_{k=1}^{n} V S_{-} \operatorname{COST}\left(V S_{k}\right)-\sum_{l=1}^{m}\left(N Q G_{l}-1\right) V S_{-} \operatorname{COST}\left(V S_{l}^{\prime}\right) \text {. }
$$

The second term in Equation 6 reflects the fact that the processing cost related to using the intermediate result should be accounted for only once due to the strategy of multiple query processing. VS_COST is evaluated by a backward recursion.

We now wish to develop the heuristic cost estimate $\hat{h}(\omega)$ and prove its admissibility. We need to define new functions. For a given access plan $\left\langle J_{P_{2}}\left(V_{1}, V_{2}\right)\right.$, $\left.J_{P_{4}}\left(V_{3}, V_{4}\right), \ldots, J_{P_{2 n}}\left(V_{2 n-1}, V_{2 n}\right)\right\rangle$ of a query $Q T_{k}^{r_{k}}$, let us define a function $\delta_{k}^{I}\left(V S_{i}\right)$ such that

\footnotetext{
${ }^{3}$ We mean by the original queries those supplied as input to MTP.
} 
$\delta_{k}^{\prime}\left(V S_{i}\right)$ returns the remaining cost starting from the view $V S_{i}$ along the individual access plan of $Q T_{k}^{r_{k}}$ to get the result of $Q T_{k}^{r_{k}}$. Here,

$$
\delta_{k}^{l}\left(V S_{i}\right)=V S_{-} \operatorname{COST}\left(V I E W\left(Q T_{k}^{r_{k}}\right)\right)-V S_{-} \operatorname{COST}\left(V S_{i}\right),
$$

where the superscript $I$ of $\delta_{k}^{\prime}$ indicates that the processing cost is evaluated along the access plan which is determined by an individual distributed query processing strategy. We define the function $\Gamma$ as follows:

$$
\begin{aligned}
\Gamma\left(V S_{1}, V S_{2}\right)= & \left\{\left(V_{k}, S_{k}\right) \mid \exists J_{P_{k}}\left(\left(V_{k}=J_{P_{k}}\left(V_{1}, V_{2}\right)\right) \wedge\left(V_{1}, S_{1}\right) \in V S_{1}\right.\right. \\
& \left.\left.\wedge\left(V_{2}, S_{2}\right) \in V S_{2}\right)\right\} .
\end{aligned}
$$

Let $x=(V, S)$ indicate a view $V$ stored at site $S$. Let $\delta^{l}(x)$ denote the remaining processing cost of an individual query access plan starting from the view $V$. Let $\hat{\delta}\left(V S_{k}\right)$ denote the estimate of the remaining processing cost associated with a current view $V S_{k}$. Then,

$$
\begin{aligned}
& \hat{\delta}\left(V S_{k}\right)=\sum_{y \in V S^{\prime}}\left\{\min _{x \in \Gamma\left(V S_{k}, y\right) \wedge S^{\prime \prime} \in S}\left\{\operatorname{cst}\left(\left\{V S_{k,} y\right\},\left(V, S^{\prime \prime}\right)\right)\right\}\right. \\
& \left.+\max _{x \in I\left(V S_{k}, y\right) \wedge S^{\prime \prime} \in S}\left\{\delta^{\prime}(x)-\psi_{s s^{\prime \prime}}^{c}(V)\right\}\right\} ;
\end{aligned}
$$

where $S$ is the set of all sites in the given computer network; VS $=$ $\left.\left\{\left(V_{l}, S_{l}\right) \mid \exists J_{P_{l}}\left(J_{p_{l}}\left(V_{k}, V_{l}\right)=V_{i}\right) \wedge\left(V_{i}, S_{i}\right) \in \Gamma\left(V_{k}, V_{l}\right) \wedge S_{i}, S_{l} \in S\right)\right\}$.

Let CVS be the set of all the current views in state $\omega$. Then,

$$
\hat{h}(\omega)=\sum_{v s \in C v s} \hat{\delta}(V S) .
$$

We prove the admissibility of $\hat{h}$ below.

Theorem 1 Suppose that the individual access plan of each query is optimal. Then $h(\omega) \geqslant \hat{h}(\omega)$ where $h(\omega)$ is the minimal cost to reach the goal state $\omega_{g}$ from the current state $\omega$.

Proof. Without loss of generality, let

$$
\begin{aligned}
\hat{h}(\omega)= & \sum_{y \in V S^{\prime}}\left\{\min _{x \in \Gamma\left(V S_{k}, y\right) \wedge S^{\prime \prime} \in S}\left\{\operatorname{cst}\left(\left\{V S_{k}, y\right\},\left(V, S^{\prime \prime}\right)\right)\right\}\right. \\
& \left.+\max _{x \in \Gamma\left(V S_{k}, y\right) \wedge S^{\prime \prime} \in S}\left\{\delta^{\prime}(x)-\psi_{s S^{\prime \prime}}^{c}(V)\right\}\right\} .
\end{aligned}
$$

We make the assumption that there exists only one current $V S_{k}$ and only one $y$. The situation is shown in Fig. 7. In Fig. 7, $\Gamma\left(V S_{k}, y\right)=\left\{\left(V_{i}, S_{i}\right) \mid i=1,2, \ldots, n\right\}, S=\left\{S_{1}, S_{2}, \ldots, S_{s}\right\}$ and $V S^{\prime}=\{y\}$.

Suppose that we generate a superquery $Q G$ starting from $\left(V_{k}, S_{k}\right)$ such that all the results of queries $Q T_{k}^{r_{k}}$ for $k=1,2, \ldots, n$ can be obtained by accessing the result of $Q G$. Furthermore, let us assume that the access plan of $Q G$ is optimal among all the feasible access plans of superqueries. As shown in Fig. 7, let us assume that $\left(V^{\prime}, S^{\prime}\right)$ is the view in the access plan of $Q G$ from which the views $\left(V_{i}, S_{i}\right)$ for $i=1,2, \ldots, n$ can be obtained. Let $\delta^{\prime}\left(\left(V^{\prime}, S^{\prime}\right)\right)$ be the remaining processing cost of $Q G$ starting from $\left(V^{\prime}, S^{\prime}\right)$. Since each individual access plan of queries is assumed to be optimal, we know that $\psi_{s s^{\prime}}^{c}\left(V_{i}\right)+$ $\delta^{\prime}\left(\left(V_{i}, S^{\prime}\right)\right)>\delta_{i}^{\prime}\left(\left(V_{i}, S_{i}\right)\right)$ for all $i=1,2, \ldots n$. Otherwise, it leads to a contradiction since 


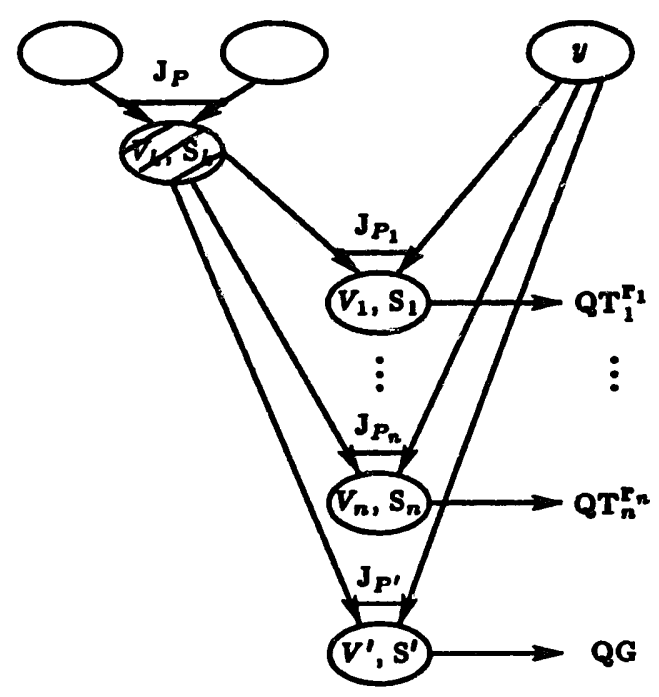

Fig. 7. A state with only one current view.

$\delta_{i}^{l}\left(\left(V_{i}, S_{i}\right)\right)$ does not become minimal in such a case. Since $\operatorname{VOL}\left(V^{\prime}\right) \geqslant \operatorname{VOL}\left(V_{i}\right)$, $\delta^{\prime}\left(\left(V^{\prime}, S^{\prime}\right)\right) \geqslant \delta^{\prime}\left(\left(V_{i}, S^{\prime}\right)\right)>\delta_{i}^{\prime}\left(\left(V_{i}, S_{i}\right)\right)-\psi_{s s^{\prime}}^{c}\left(V_{i}\right)$ for any site $S^{\prime} \in S$ and $i=1,2, \ldots, n$. Thus, $\delta^{\prime}\left(\left(V^{\prime}, S^{\prime}\right)\right)>\max _{x \in \Gamma\left(V s_{k}, y\right) \wedge S^{\prime \prime} \in S}\left\{\delta^{\prime}(x)-\psi_{s s^{\prime \prime}}^{c}(V)\right\}$.

Now, let us consider all the possible ways of constructing $V_{i}$ for $i=1,2, \ldots, n$ from the views $V S_{k}$ and $y$. For each $V_{i}$, we have $s$ ways of constructing it where $s$ is the number of sites. Let the view $\left(V^{\prime \prime}, S^{\prime \prime}\right)$ be the view such that $\left(V^{\prime \prime}, S^{\prime \prime}\right) \in\left\{\left(V_{i}, S_{i}\right) \mid i=1,2, \ldots, n\right\}$ and $\operatorname{cst}\left(\left\{V S_{k}, y\right\},\left(V^{\prime \prime}, S^{\prime \prime}\right)\right)$ is minimal. Since $V O L\left(V^{\prime}\right) \geqslant V O L\left(V^{\prime \prime}\right)$, we know that $\operatorname{cst}\left(\left\{V S_{k}, y\right\}\right.$, $\left.\left(V^{\prime}, S^{\prime}\right)\right) \geqslant \operatorname{cst}\left(\left\{V S_{k}, y\right\},\left(V^{\prime \prime}, S^{\prime}\right)\right) \geqslant \operatorname{cst}\left(\left\{V S_{k}, y\right\}\left(V^{\prime \prime}, S^{\prime \prime}\right)\right)$. In other words, $\operatorname{cst}\left(\left\{V S_{k}, y\right\}\right.$, $\left.\left(V^{\prime}, S^{\prime}\right)\right) \geqslant \min _{x \in I\left(V S_{k}, y\right) \wedge s^{\prime \prime} \in S}\left\{c s t\left(\left\{V S_{k}, y\right\},\left(V, S^{\prime \prime}\right)\right)\right\}$, where $x=(V, S)$. Thus, the lower bound of $h\left(V S_{k}\right)$ where $h\left(V S_{k}\right)$ is the remaining processing cost associated with the current view $V S_{k}$ when an optimal superquery is generated, is

$$
\begin{aligned}
\hat{h}\left(V S_{k}\right)= & \left\{\min _{x \in \Gamma\left(V S_{k}, y\right) \wedge S^{\prime \prime} \in S}\left\{\operatorname{cst}\left(\left\{V S_{k}, y\right\},\left(V, S^{\prime \prime}\right)\right)\right\}\right. \\
& +\max _{x \in \Gamma\left(V S_{k}, y\right) \wedge S^{\prime \prime} \in S}\left\{\delta^{\prime}(x)-\psi_{S S^{\prime \prime}}^{c}(V)\right\} \square
\end{aligned}
$$

It is easily shown that $\hat{\delta}\left(V S_{k}\right)$ is simplified to $\delta_{k}^{l}\left(V S_{k}\right)$ when $V S_{k}$ is the current view of $Q T_{k}^{r_{k}}$ which is marked by the activation of generation rule $I$-rule. This is summarized below:

$$
\hat{\delta}\left(V S_{k}\right)= \begin{cases}\delta_{k}^{I}\left(V S_{k}\right) & \text { if } \operatorname{CARD}\left(\Gamma\left(V S_{k}, y\right)\right)=1 \\ \text { Eq. }(9) & \text { otherwise }\end{cases}
$$

where CARD denotes the cardinality of a set.

Finally, we wish to describe the test rule. For each search step, we evaluate $\hat{f}(\omega)=$ $g(\omega)+\hat{h}(\omega)$. Among all the siates which are generated at previous search steps, but are not expanded, or which are generated at the current search step, we select the state which has the minimal cost estimate $\hat{f}(\omega)$, and we test that state whether it is a goal state or not. If that state is found to be a goal state, then the search process is stopped. Otherwise, we expand that state, and the search process resumes. 


\subsection{A special case}

In this section we present heuristic cost evaluation functions for a simple case in which the access plans of the given queries comply with the following assumption, called the assumption of fixed access order: (i) for an access step $J_{p}\left(V_{1}, V_{2}\right)$, the join is performed at the site where $V_{2}$ is located by moving $V_{1}$ there; (ii) the order of the access step in the access sequence of a newly generated superquery precisely follows those of the original queries which use the (intermediate) result of that new superquery. ${ }^{4}$ Due to this assumption, the site information is implicitly ignored throughout this section.

Here, we do not assume that each individual access plan is optimal. Under the assumption of fixed access order, it is generally not feasible to infer subquery relationships in the middle of the access sequences of two different queries. ${ }^{5}$ Hence, we can assume that all the superquery relationships which would be profitable can be inferred from the query graph shown in Fig. 8. The function $g$ is defined as in Equation 6.

For $\hat{h}$, we need to define the two functions $\hat{\rho}$ and $\Gamma$. Let $V_{1}$ and $V_{2}$ be sets of views. $\Gamma$ is defined as follows:

$$
\Gamma\left(V_{1}, V_{2}\right)=\left\{V \mid \exists J_{P_{k}}\left(\left(V=J_{P_{k}}\left(V_{1}, V_{2}\right)\right) \wedge\left(V_{1} \in V_{1}\right) \wedge\left(V_{2} \in V_{2}\right)\right)\right\}
$$

For a set $V$ of views, the function $\hat{\rho}(V)$ optimistically estimates the sum of the remaining costs of queries which use the intermediate results in $V$.

Let $x, y$ and $z$ denote views (see Fig. 8). Then,

$$
\hat{\rho}(V)=\sum_{y \in V^{\prime}}\left\{\max _{(x \in V) \wedge(z \in \Gamma(x, y))}\{\operatorname{cst}((x, y), z)\}+\hat{\rho}(\Gamma(V, y))\right\},
$$

where $V^{\prime}=\left\{V^{\prime} \mid \exists J_{P_{k}}\left(\left(J_{P_{k}}\left(V, V^{\prime}\right)=V^{\prime \prime}\right) \wedge(V \in V) \wedge\left(V^{\prime \prime} \in \Gamma\left(V, V^{\prime}\right)\right)\right)\right\} . \hat{\rho}(V)$ is evaluated by

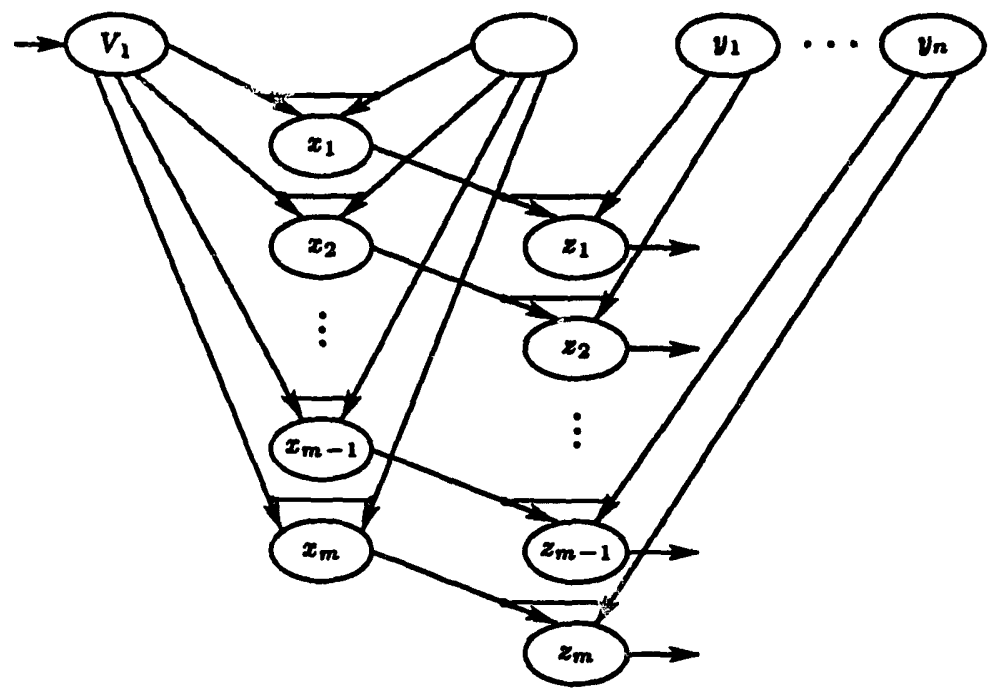

Fig. 8. A fr Jle query graph.

\footnotetext{
${ }^{4}$ This assumption is more likely to be valid in a centralized environment, but it may also be applicable to local area networks.

${ }^{5}$ Refer to [25].
} 
a forward recursion. The boundary conditions are as follows:

Case 1. When $V_{3}=J_{P}\left(V_{1}, V_{2}\right), \hat{\rho}\left(V_{1}\right)=\operatorname{cst}\left(\left(V_{1}, V_{2}\right), V_{3}\right)$.

Case 2. When $V_{2}=u \operatorname{uop}\left(V_{1}\right), \hat{\rho}\left(V_{1}\right)=\operatorname{cst}\left(V_{1}, V_{2}\right)$.

Case 3. When $\hat{\rho}(V)=\operatorname{cst}\left(V, V_{f}\right), \hat{\rho}(V)=0$.

Now, let $\boldsymbol{C V}$ be the set of all the current views in state $\omega$. The heuristic cost evaluation function $\hat{h}$ is defined as follows:

$$
\hat{h}(\omega)=\sum_{V \in C V} \hat{\rho}(V) \text {. }
$$

The initial state $\omega_{0}$ is $\left(\boldsymbol{G}, \boldsymbol{A P}, \boldsymbol{C A S}, 0, \hat{h}\left(\omega_{0}\right)\right)$ where $\boldsymbol{A P}$ is the set of access plans which are not reformulated, and $C A S$ is the empty set. The goal state $\omega_{g}$ is $\left(G, A P, C A S, g\left(\omega_{g}\right), 0\right)$ where $G$ and $A P$ are the reformulated query graph and the set of reformulated access plans, respectively; $C A S$ is $\left\{V_{f}\right\}$.

We prove the admissibility of $\hat{h}$ below.

Theorem 2. $h(\omega) \geqslant \hat{h}(\omega)$ where $h(\omega)$ is the minimal cost from the current state $\omega$ to the goal state $\omega_{g}$.

Proof. Let $V_{i}$ for $i=1,2,3,4$ denote views. Let $J_{P_{1}}\left(V_{1}, V_{2}\right)$ be an access step chosen for the evaluation of some $\hat{\rho}(V)$ defined in Equation 13 where $V$ is a current view of a query at state $\omega$. Let $\rho(V)$ be an optimal remaining processing cost, at state $\omega$, for the current view $V$ using the multiple query processing strategy. (Equation (13) is clearly true if the individual access plan are used.) For the access step $J_{P_{1}}\left(V_{1}, V_{2}\right)$ of the query, let the corresponding access step of the superquery be $J_{P_{3}}\left(V_{3}, V_{4}\right)$ which is involved in the construction of an optimal access plan. Let $V_{1}^{\prime}=J_{P_{1}}\left(V_{1}, V_{2}\right)$ and $V_{3}^{\prime}=J_{P_{3}}\left(V_{3}, V_{4}\right)$. Since $J_{P_{3}}\left(V_{3}, V_{4}\right)$ is an access step of the superquery, the processing cost of performing this access step is greater than or equal to any corresponding access step of the query which uses the result of execution of $J_{P_{3}}\left(V_{3}, V_{4}\right)$. Hence, $\operatorname{cst}\left(\left(V_{3}, V_{4}\right), V_{3}^{\prime}\right) \geqslant \operatorname{cst}\left(\left(V_{1}, V_{2}\right), V_{1}^{\prime}\right)$. Since $\operatorname{cst}\left(\left(V_{3}, V_{4}\right), V_{3}^{\prime}\right) \geqslant \operatorname{cst}\left(\left(V_{1}, V_{2}\right), V_{1}^{\prime}\right)$ for any access step $J_{P_{1}}\left(V_{1}, V_{2}\right)$ which comes behind the current view $V, \rho(V) \geqslant \hat{\rho}(V)$. Therefore, $h(\omega) \geqslant \Sigma_{V \in C V} \hat{\rho}(V)$.

\section{An example}

This example illustrates the operation of Multiple Transaction Processor. For simplicity, attention is restricted to the special case described in Section 4.3. The example shows how multiple queries occurring concurrently in a distributed database system can be processed optimally by utilizing jointly both database semantics and logical information of predicate conditions of queries.

The distributed database $r$ contains three relations $r_{1}, r_{2}$ and $r_{3}$ at site 1 , site 2, and site 3 , respectively, as shown in Fig. 9. Let $V_{a}=J_{P_{4} \wedge P_{5}}\left(\pi_{Y_{1}}\left(r_{1}\right), r_{2}\right)$ and $V_{b}=J_{P_{3}}\left(\pi_{Z_{1}}\left(r_{1}\right), r_{2}\right)$. Two queries $Q T_{2}$ and $Q T_{3}$ occur at site 1 with the following access plans:

$$
\begin{aligned}
& \operatorname{access\_ plan}\left(Q T_{2}\right):\left\langle J_{P_{4} \wedge P_{5}}\left(\pi_{Y_{1}}\left(r_{1}\right), r_{2}\right), J_{P_{7} \wedge P_{8}}\left(\pi_{Y_{2}}\left(V_{a}\right), r_{3}\right)\right\rangle \text {, and } \\
& \text { access_plan }\left(Q T_{3}\right):\left\langle J_{P_{3}}\left(\pi_{Z_{1}}\left(r_{1}\right), r_{2}\right), J_{P_{q}}\left(\pi_{Z_{2}}\left(V_{b}\right), r_{3}\right)\right\rangle \text {, }
\end{aligned}
$$

where $Y_{1}, Z_{1} \subseteq R_{1}, Y_{2} \subseteq R_{1} \cup R_{2}$, and $Z_{2} \subseteq R_{1} \cup R_{2}$. The access plan of $Q T_{2}$ implies that 


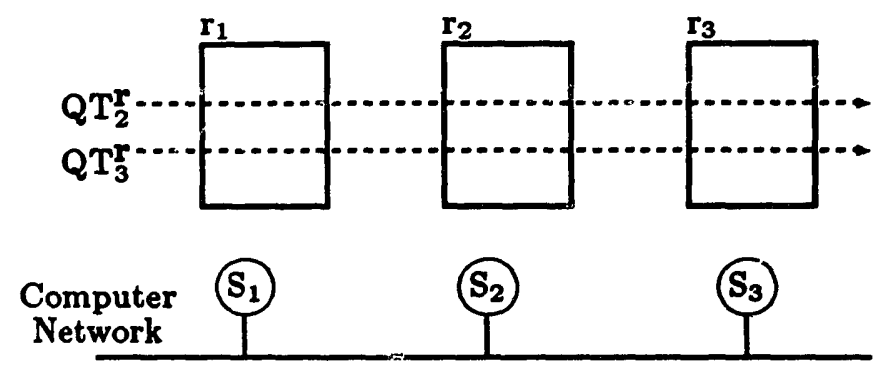

Fig. 9. Example of a distributed database with two queries.

$\pi_{Y_{1}}\left(r_{1}\right)$ is performed at site $S_{1} ; \pi_{Y_{2}}\left(J_{P_{4} \wedge P_{5}}\left(V^{\prime}, r_{2}\right)\right)$ with $V^{\prime}=\pi_{Y_{1}}\left(r_{1}\right)$ being transferred from $S_{1}$ is performed at site $S_{2} ; J_{P_{7} \wedge P_{8}}\left(V^{\prime \prime}, r_{3}\right)$ is performed at $S_{3}$ with $V^{\prime \prime}=\pi_{Y_{2}}\left(J_{P_{4} \wedge P_{5}}\left(\pi_{Y_{1}}\left(r_{1}\right), r_{2}\right)\right.$. The access plan of $Q T_{3}$ can be interpreted similarly.

During the planning step, a set of integrity constraints is inferred from logical and semantic information using inference-guiding heuristics. ${ }^{6}$ The plan step infers only relevant ICs for the efficient identification of subquery relationships and systematic generation of superqueries in the subsequent search step. The following is the list of all the relevant ICs associated with relations $r_{1}$ and $r_{2}$, and $r_{2}$ and $r_{3}$.

ICs related to $r_{1}$ and $r_{2}$

\begin{tabular}{ccc}
\hline$P_{1} \wedge P_{2}$ & $\Rightarrow$ & $P_{1} \wedge P_{2}$ \\
$P_{4} \wedge P_{5}$ & $\Rightarrow$ & $P_{1} \wedge P_{2}$ \\
$P_{1} \wedge P_{2}$ & $\Rightarrow$ & $P_{1}$ \\
$P_{4} \wedge P_{5}$ & $\Rightarrow$ & $P_{1}$ \\
$P_{3}$ & $\Rightarrow$ & $P_{1}$ \\
$P_{1} \wedge P_{2}$ & $\Rightarrow$ & $P_{2}$ \\
$P_{4} \wedge P_{5}$ & $\Rightarrow$ & $P_{2}$ \\
$P_{3}$ & $\Rightarrow$ & $P_{2}$ \\
$P_{4} \wedge P_{5}$ & $\Rightarrow$ & $P_{4} \wedge P_{5}$ \\
$P_{3}$ & $\Rightarrow$ & $P_{3}$ \\
$P_{1}$ & $\Rightarrow$ & $P_{1}$ \\
$P_{2}$ & $\Rightarrow$ & $P_{2}$ \\
\hline
\end{tabular}

ICs related to $r_{2}$ and $r_{3}$

\begin{tabular}{ccc}
\hline$P_{7}$ & $\Rightarrow$ & $P_{7}$ \\
$P_{7} \wedge P_{8}$ & $\Rightarrow$ & $P_{7} \quad c$ \\
$P_{8}$ & $\Rightarrow$ & $P_{8}$ \\
$P_{7} \wedge P_{8}$ & $\Rightarrow$ & $P_{8}$ \\
$P_{7} \wedge P_{8}$ & $\Rightarrow$ & $P_{6}$ \\
$P_{7} \wedge P_{8}$ & $\Rightarrow$ & $P_{7} \wedge P_{8}$ \\
$P_{8}$ & $\Rightarrow$ & $P_{6}$ \\
$P_{9}$ & $\Rightarrow$ & $P_{9}$ \\
$P_{7}$ & $\Rightarrow$ & $P_{11}$ \\
$P_{7} \wedge P_{8}$ & $\Rightarrow$ & $P_{10}$ \\
$P_{8}$ & $\Rightarrow$ & $P_{10}$ \\
$P_{10}$ & $\Rightarrow$ & $P_{10}$ \\
\hline
\end{tabular}

The initial state for this example is shown in Fig. 10 where the access plans of $Q T_{2}$ and $Q T_{3}$ are integrated.

The processing costs for $Q T_{2}$ and $Q T_{3}$ are described below when individual distributed query processing strategies are used.

$$
\begin{aligned}
Q T_{-} \operatorname{COST} T_{2}= & \psi^{\prime}\left(\pi_{Y_{1}}, r_{1}\right)+\psi^{c}\left(\pi_{Y_{1}}\left(r_{1}\right)\right)+\psi^{\prime}\left(J_{P_{4} \wedge P_{5}}, \pi_{Y_{1}}\left(r_{1}\right), r_{2}\right) \\
& +\psi^{l}\left(\pi_{Y_{2}}, V_{a}\right)+\psi^{c}\left(\pi_{Y_{2}}\left(V_{a}\right)\right)+\psi^{l}\left(J_{P_{7} \wedge P_{8}}, \pi_{Y_{2}}\left(V_{a}\right), r_{3}\right) \\
Q T_{-} C O S T_{3}= & \psi^{\prime}\left(\pi_{Z_{1}}, r_{1}\right)+\psi^{c}\left(\pi_{Z_{1}}\left(r_{1}\right)\right)+\psi^{\prime}\left(J_{P_{3}}, \pi_{Z_{1}}\left(r_{1}\right), r_{2}\right) \\
& +\psi^{\prime}\left(\pi_{Z_{2}}, V_{b}\right)+\psi^{c}\left(\pi_{Z_{2}}\left(V_{b}\right)\right)+\psi^{l}\left(J_{P_{9}}, \pi_{Z_{2}}\left(V_{b}\right), r_{3}\right) .
\end{aligned}
$$

\footnotetext{
"The planning technique is described in detail in [25].
} 


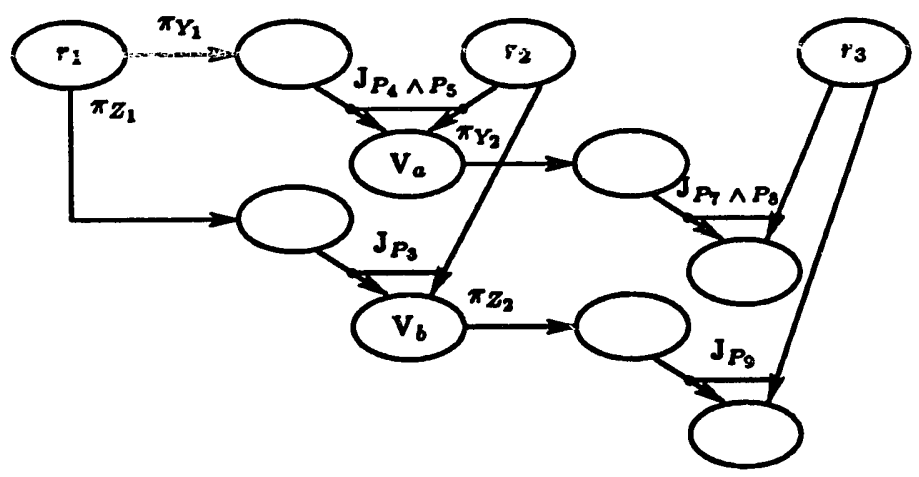

Fig. 10. A graphical representation of the initial state.

All site information is ignored here since we assume that all the sites have the same processing capability and that the communication cost does not depend on sites. In Equation 15, $\quad \operatorname{cst}_{2}\left(\left(\pi_{Y_{1}}\left(r_{1}\right), r_{2}\right), V_{a}\right)=\psi^{l}\left(\pi_{Y_{1}}, r_{1}\right)+\psi^{c}\left(\pi_{Y_{1}}\left(r_{1}\right)\right)+\psi^{l}\left(J_{P_{4} \wedge P_{5}}, \pi_{Y_{1}}\left(r_{1}\right), r_{2}\right)$ which is associated with the intersite join operation $J_{P_{4} \wedge P_{5}}$, and $\operatorname{cst}_{2}\left(\left(\pi_{Y_{2}}\left(V_{a}\right), r_{3}\right)\right.$, $\left.J_{P_{7} \wedge P_{8}}\left(\pi_{Y_{2}}\left(V_{a}\right), r_{3}\right)\right)=\psi^{\prime}\left(\pi_{Y_{2}}, J_{P_{4} \wedge P_{5}}\left(\pi_{Y_{1}}\left(r_{1}\right), r_{2}\right)\right)+\psi^{c}\left(\pi_{Y_{2}}\left(J_{P_{4} \wedge P_{5}}\left(\pi_{Y_{1}}\left(r_{1}\right), r_{2}\right)\right)\right)+\psi^{l}\left(J_{P_{7} \wedge P_{8}}\right.$, $\left.\pi_{Y_{2}}\left(J_{P_{4} \wedge P_{5}}\left(\pi_{Y_{1}}\left(r_{1}\right), r_{2}\right)\right), r_{3}\right)$, which is related to the intersite join operation $J_{P_{7} \wedge P_{8}}$.

We first discuss the search process. For example, by employing the two promising ICs $P_{3} \Rightarrow P_{1}$ and $P_{4} \wedge P_{5} \Rightarrow P_{1}$, state 1 , which is shown in Fig. 11, is generated from the initial state. In Fig. 11, the current access step of the query $Q G_{1}$ is $J_{P_{1}}\left(\pi_{Y_{1} \cup Z_{1}}\left(r_{1}\right), r_{2}\right)$, and the current view of the query is $V_{1}$. We make the assumption that the attributes involved in the join operation $J_{P_{1}}$ are contained in $Y_{1} \cup Z_{1}$.

The construction rule for the join operation permits two intersite join operations $J_{P_{4} \wedge P_{5}}$ and $J_{P_{3}}$ to be replaced by one intersite join $J_{P_{1}}$. Here, the superquery $Q G_{1}$ of both $Q T_{2}$ and $Q T_{3}$ is generated with respect to $\left\langle r_{1}, r_{2}\right\rangle$. The view $V_{1}$ which is the result of the superquery execution allows for the production of intermediate results $V_{a}$ and $V_{b}$ of $Q T_{2}$ and $Q T_{3}$ since $V_{a}=\pi_{Y_{1} \cup R_{2}}\left(\sigma_{P_{4} \wedge P_{5}}\left(V_{1}\right)\right)$ and $V_{b}=\pi_{Z_{1} \cup R_{2}}\left(\sigma_{P_{3}}\left(V_{1}\right)\right)$.

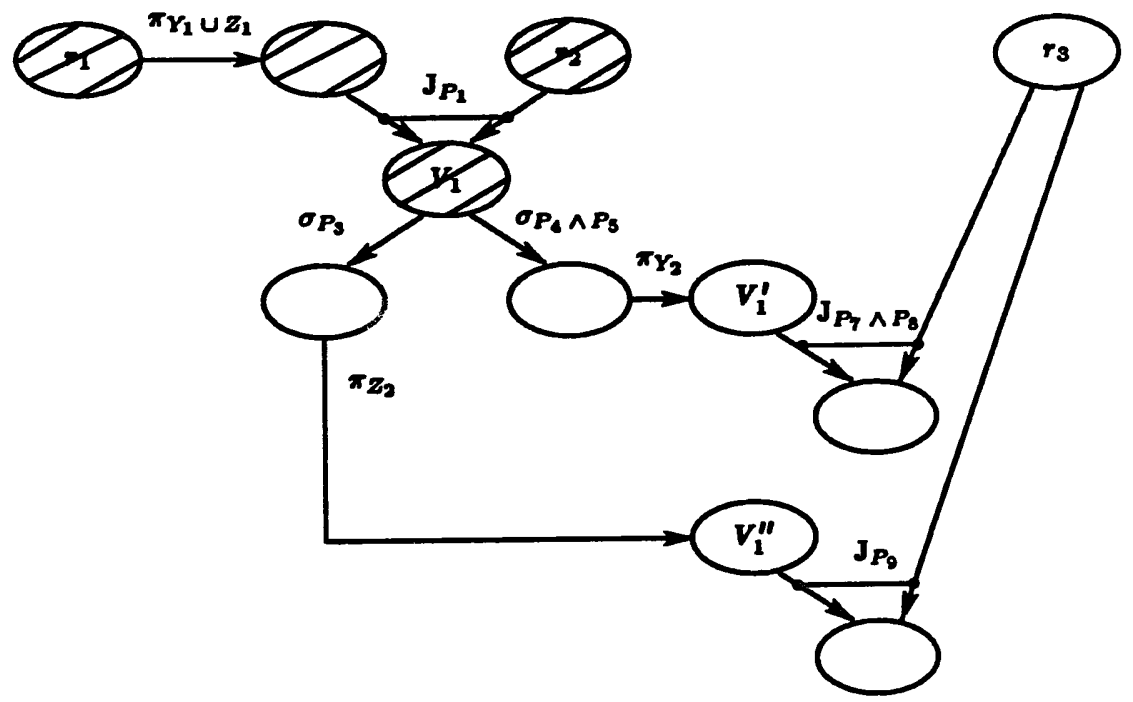

Fig. 11. A graphical representation of state 1 . 
State 1 is generated by the activation of $M$-rule, and state 4 which is shown in Fig. 12 is generated by the activation of $I$-rule. All the views which are utilized in the search step are marked by /// in both Figs 11 and 12 . Note that state 1 is generated following the access sequence of execution plans of both $Q T_{2}$ and $Q T_{3}$.

$g(\omega)$ is the processing cost incurred at the current state $\omega$. Let us define $\hat{h}_{l}(\omega)$ as the heuristic cost evaluation function which estimates the sum of the incremental remaining processing cost for each query, from the current access step to the final access step, when the individual distributed query processing strategies are used. $h(\omega)$ is the minimal incremental cost from the current state $\omega$ which can occur by reformulating access plans in the context of multiple query processing. As before, let $\hat{h}(\omega)$ be an optimistic estimate of $h(\omega)$.

Let $V_{1}^{\prime}=\pi_{Y_{2}}\left(\sigma_{P_{4} \wedge P_{5}}\left(V_{1}\right)\right)$, and $V_{1}^{\prime \prime}=\pi_{Z_{2}}\left(\sigma_{P_{3}}\left(V_{1}\right)\right)$ as shown in Fig. 11. Also, let $\omega$ be state 1 .

$$
\begin{aligned}
g(\omega)= & Q G_{-} \operatorname{COST} T_{1}\left(r_{1}, r_{2}\right) \\
= & \operatorname{cst}\left(\left(\pi_{Y_{1} \cup Z_{1}}\left(r_{1}\right), r_{2}\right), V_{1}\right) \\
= & \psi^{\prime}\left(\pi_{Y_{1} \cup Z_{1}}, r_{1}\right)+\psi^{c}\left(\pi_{Y_{1} \cup Z_{1}}\left(r_{1}\right)\right)+\psi^{\prime}\left(J_{P_{1}}, \pi_{Y_{1} \cup Z_{1}}\left(r_{1}\right), r_{2}\right) \\
\hat{h}_{l}(\omega)= & \operatorname{cst}_{2}\left(\left(V_{1}^{\prime}, r_{3}\right), J_{P_{7} \wedge P_{8}}\left(V_{1}^{\prime}, r_{3}\right)\right) \\
& +\operatorname{cst}_{3}\left(\left(V_{1}^{\prime \prime}, r_{3}\right), J_{P_{9}}\left(V_{1}^{\prime \prime}, r_{3}\right)\right) .
\end{aligned}
$$

$\operatorname{cst}\left(\left(\pi_{Y_{1} \cup Z_{1}}\left(r_{1}\right), r_{2}\right), V_{1}\right)$ denotes the processing cost of the current access step of the superquery $Q G_{1}$. The access step $J_{P_{1}}\left(\pi_{Y_{1} \cup Z_{1}}\left(r_{1}\right), r_{2}\right)$ is the current access step for all the queries $Q T_{2}, Q T_{3}$ and $Q G_{1}$ at the state shown in Fig. 11. cst $_{2}$ denotes the incremental cost incurred using the intermediate result $V_{1}$ to get the answer of the query $Q T_{2}$. Similarly, cst denotes that for the query $Q T_{3}$, using the same intermediate result $V_{1}$. Here,

$$
\begin{aligned}
\operatorname{cst}_{2}\left(\left(V_{1}^{\prime}, r_{3}\right), J_{P_{7} \wedge P_{8}}\left(V_{1}^{\prime}, r_{3}\right)\right)= & \psi^{\prime}\left(\sigma_{P_{4} \wedge P_{5}}, V_{1}\right)+\psi^{\prime}\left(\pi_{Y_{2}}, \sigma_{P_{4} \wedge P_{5}}\left(V_{1}\right)\right) \\
& +\psi^{c}\left(V_{1}^{\prime}\right)+\psi^{\prime}\left(J_{P_{7} \wedge P_{8}}, V_{1}^{\prime}, r_{3}\right) \\
\operatorname{cst}_{3}\left(\left(V_{1}^{\prime \prime}, r_{3}\right), J_{P_{9}}\left(\left(V_{1}^{\prime \prime}\right), r_{3}\right)\right)= & \psi^{\prime}\left(\sigma_{P_{3}}, V_{1}\right)+\psi^{\prime}\left(\pi_{Z_{2}}, \sigma_{P_{3}}\left(V_{1}\right)\right) \\
& +\psi^{c}\left(V_{1}^{\prime \prime}\right)+\psi^{l}\left(J_{P_{9}}, V_{1}^{\prime \prime}, r_{3}\right) .
\end{aligned}
$$

Now, we attempt to extend the superquery relationship since it may reduce the total processing cost further. Since we do not know the existence of ICs at state 1, we do an optimistic conjecture that either $P_{9} \Rightarrow P_{7} \wedge P_{8}$ or $P_{7} \wedge P_{8} \Rightarrow P_{9}$ or both might be true. This

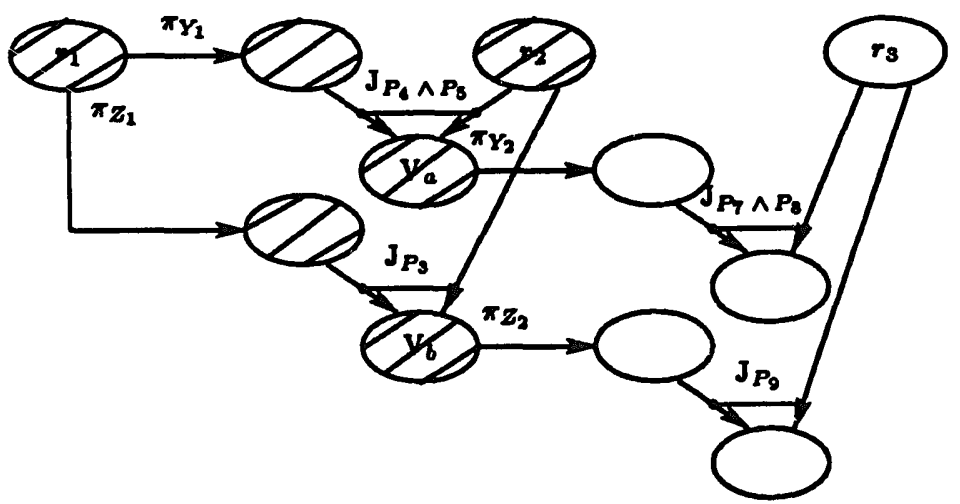

Fig. 12. A graphical representation of state 4 . 
optimistic conjecture allows us to estimate the remaining cost estimate $\hat{h}(\omega)$ at state 1 . Using the construction rule for the join operation, the feasible state generated from state 1 which is based on the optimistic conjecture is depicted in Fig. 13.

In the process of extending the superquery with respect to $\left\langle r_{1}, r_{2}\right\rangle$ in Fig. 11 to that with respect to $\left\langle r_{1}, r_{2}, r_{3}\right\rangle$ in Fig. 13, the local selection operations $\sigma_{P_{3}}$ and $\sigma_{P_{4} \wedge P_{5}}$ are postponed to the next stage in the access sequence, and a new projection operation $\pi_{Z}$ is added. $Z=Z_{2} \cup Y_{2} \cup X$ where $X$ denotes the attributes involved in the selection operations $\sigma_{P_{3}}$ and $\sigma_{P_{4} \wedge P_{5}}$. Here, we can define two cost estimates for the remaining cost; one is $\hat{h}_{1}(\omega)$ based on the optimistic conjecture $P_{7} \wedge P_{8} \Rightarrow J_{P_{9}}$, and the other $\hat{h}_{2}(\omega)$ based on $J_{P_{9}} \Rightarrow P_{7} \wedge P_{8}$. These estimates are evaluated as follows:

$$
\begin{aligned}
& \hat{h}_{1}(\omega)=\operatorname{cst}\left(\left(\pi_{Z}\left(V_{1}\right) r_{3}\right), V_{2}\right)+\delta_{I}(\omega) \\
& \hat{h}_{2}(\omega)=\operatorname{cst}\left(\left(\pi_{Z}\left(V_{1}\right) r_{3}\right), V_{2}^{\prime}\right)+\delta_{I}^{\prime}(\omega),
\end{aligned}
$$

where $V_{2}^{\prime}=J_{P_{7} \wedge P_{8}}\left(\pi_{Z}\left(V_{1}\right), r_{3}\right) ; \delta_{1}$ and $\delta_{1}^{\prime}$ account for the local processing cost at the final access step to get the result of each query from those of superqueries. Here,

$$
\begin{aligned}
\operatorname{cst}\left(\left(\pi_{Z}\left(V_{1}\right) r_{3}\right), V_{2}\right)= & \psi^{l}\left(\pi_{Z}, V_{1}\right)+\psi^{c}\left(\pi_{Z}\left(V_{1}\right)\right)+\psi^{l}\left(J_{P_{q}}, \pi_{Z}\left(V_{1}\right), r_{3}\right) \\
\operatorname{cst}\left(\left(\pi_{Z}\left(V_{1}\right) r_{3}\right), V_{2}^{\prime}\right)= & \psi^{l}\left(\pi_{Z}, V_{1}\right)+\psi^{c}\left(\pi_{Z}\left(V_{1}\right)\right)+\psi^{l}\left(J_{P_{7} \wedge P_{8}}, \pi_{Z}\left(V_{1}\right), r_{3}\right) \\
\delta_{l}(\omega)= & \psi^{l}\left(\sigma_{P_{3}}, V_{2}\right)+\psi^{l}\left(\pi_{Z_{2} \cup R_{3}}, \sigma_{P_{3}}\left(V_{2}\right)\right)+ \\
& \psi^{l}\left(\sigma_{P_{4} \wedge P_{5} \wedge P_{7} \wedge P_{x}}, V_{2}\right)+\psi^{l}\left(\pi_{Y_{2} \cup R_{3}}, \sigma_{P_{4} \wedge P_{5} \wedge P_{7} \wedge P_{8}}\left(V_{2}\right)\right) \\
\delta_{I}^{\prime}(\omega)= & \psi^{l}\left(\phi_{P_{3} \wedge P_{9}}, V_{2}^{\prime}\right)+\psi^{l}\left(\pi_{Z_{2} \cup R_{3}}, \sigma_{P_{3} \wedge P_{9}}\left(V_{2}^{\prime}\right)\right)+ \\
& \psi^{l}\left(\sigma_{P_{4} \wedge P_{5}}, V_{2}^{\prime}\right)+\psi^{l}\left(\pi_{Y_{2} \cup R_{3}}, \sigma_{P_{4} \wedge P_{5}}\left(V_{2}^{\prime}\right)\right)
\end{aligned}
$$

As described before, we take $\hat{h}(\omega)=\min \left\{\hat{h}_{I}(\omega), \hat{h}_{1}(\omega), \hat{h}_{2}(\omega)\right\}$.

Now, we illustrate the search process with the actual input data given in Table 1 . We assume that the local processing costs due to projection and selection operations are

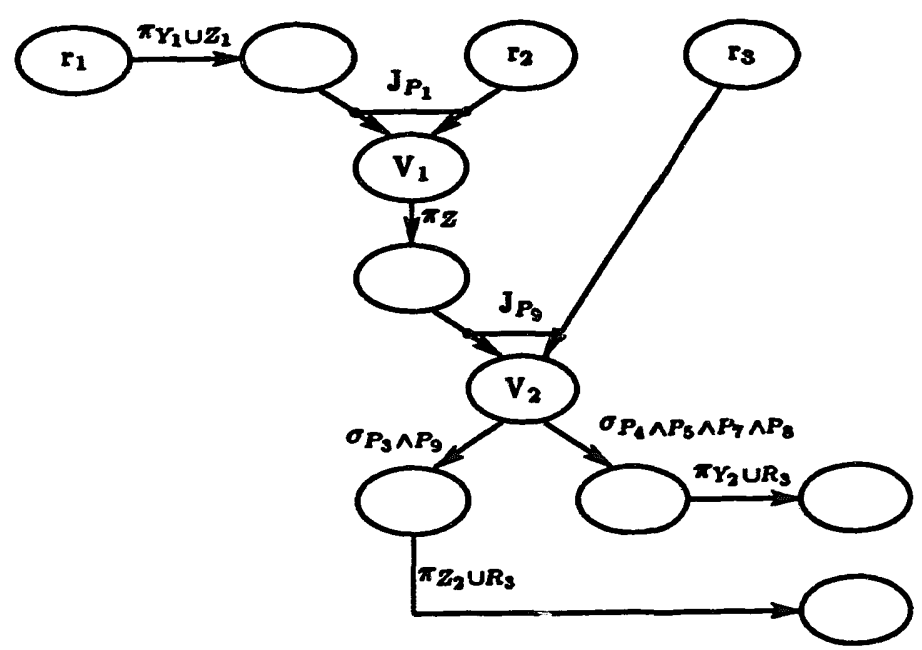

Fig. 13. A query graph based on an optimistic conjecture at state 1. 
Table 1.

The input data.

\begin{tabular}{cc|cc}
\hline & $\psi^{c}(\cdot)$ & & $\psi^{\prime}(\cdot, \cdot \cdot)$ \\
\hline$\pi_{Y_{1}}\left(r_{1}\right)$ & 5 & $\left(J_{\left.P_{4} \wedge P_{5}, \pi_{Y_{1}}\left(r_{1}\right), r_{2}\right)}\right.$ & 5 \\
$\pi_{Z_{1}}\left(r_{1}\right)$ & 5 & $\left(J_{P_{3}}, \pi_{Z_{1}}\left(r_{1}\right), r_{2}\right)$ & 5 \\
$\pi_{Y_{1} \cup Z_{1}}\left(r_{1}\right)$ & 5 & $\left(J_{P_{1}}, \pi_{Y_{1} \cup Z_{1}}\left(r_{1}\right), r_{2}\right)$ & 6 \\
- & - & $\left(J_{P_{2}}, \pi_{Y_{1} \cup Z_{1}}\left(r_{1}\right), r_{2}\right)$ & 9 \\
- & - & $\left(C, \pi_{Y_{1} \cup Z_{1}}\left(r_{1}\right), r_{2}\right)$ & 12 \\
$\pi_{Y_{2}}\left(V_{a}\right)$ & 2 & $\left(J_{\left.P_{1} \wedge P_{s}, \pi_{Y_{2}}\left(V_{a}\right), r_{3}\right)}\right)$ & 3 \\
$\pi_{Z_{2}}\left(V_{b}\right)$ & 2 & $\left(J_{P_{4}}, \pi_{Z_{2}}\left(V_{b}\right), r_{3}\right)$ & 3 \\
$\pi_{Z}\left(V_{1}\right)$ & 3 & $\left(J_{P_{10}}, \pi_{Z}\left(V_{1}\right), r_{3}\right)$ & 4 \\
\hline
\end{tabular}

negligible in comparison with intersite data transmission and join operations.

The search tree generated by MTP is shown in Fig. 14. The costs are listed in Table 2 for states $\mathrm{G}$ to 6.

In Fig. 14, the value of $\hat{f}$ for each state is circled on the upper-right corner of the box representing the state, and the uncircled number on the upper-left corner is the state number; in this example, the state numbers also indicate the order in which states are expanded, except for states $4,5,6$, and 7 which are never expanded. The darkened line shows the search path leading to the optimal solution. State 3 is generated by employing ICs $P_{7} \wedge P_{8} \Rightarrow P_{10}$ and $P_{9} \Rightarrow P_{10}$ to state 1 ; state 2 by utilizing the individual distributed query processing strategies at state 1 . State 5 corresponds to the application of individual distributed processing to the initial state without employing the knowledge available. State 6 is generated by using $P_{4} \wedge P_{5} \Rightarrow P_{2}$ and $P_{3} \Rightarrow P_{2}$; state 4 by using $P_{4} \wedge P_{5} \Rightarrow \Lambda$ and $P_{3} \Rightarrow \Lambda$. These states are never expanded since the heuristic evaluation function always underestimates the total remaining cost. In this way, many subtrees can be pruned off in more complex examples. State 5 is not expanded into states using any ICs associated with the access steps $J_{P_{7} \wedge P_{8}}\left(\pi_{Y 2}\left(V_{a}\right), r_{3}\right)$ and $J_{P_{9}}\left(\pi_{Z_{2}}\left(V_{b}\right), r_{3}\right)$, even though there are several ICs available like $P_{7} \wedge P_{8} \Rightarrow P_{10}$ and $P_{9} \Rightarrow P_{10}$. This is because, once a join operation is carried out, it is generally not possible to infer any superquery relationships on the following access step.

Among the nodes expanded from the initial state, state 1 has the minimal processing cost of 17. It is expanded, generating states 2 and 3 . Among states $2,3,4,5$, and 6 , state 3 has the minimal cost. Since state 3 cannot be expanded further, we conclude that state 3 is the goal state with the total processing cost of 19 . States 4,5 and 6 are never expanded. If state 5 were expanded, we would have state 7 as shown by the dotted line in Fig. 14. This state corresponds to the state when individual distributed query processing strategies are employed. It is easily found that the total processing cost of state 7 is equal to that of state 5; $T_{-} C O S T=Q T_{-} C O S T_{2}+Q T_{-} C O S T_{3}=\hat{f}\left(\omega_{5}\right)=30$. The savings in the total processing cost due to multiple query processing strategy amounts to $11(30-19=11)$ (neglecting the overhead due to MTP).

Table 2

The cost estimates

\begin{tabular}{rrrrrrrr}
\hline & $\omega_{0}$ & $\omega_{1}$ & $\omega_{2}$ & $\omega_{3}$ & $\omega_{4}$ & $\omega_{5}$ & $\omega_{6}$ \\
\hline$g$ & 0 & 12 & 22 & 19 & 18 & 20 & 15 \\
$\hat{h}$ & 15 & 5 & 0 & 0 & 5 & 10 & 5 \\
\hline
\end{tabular}




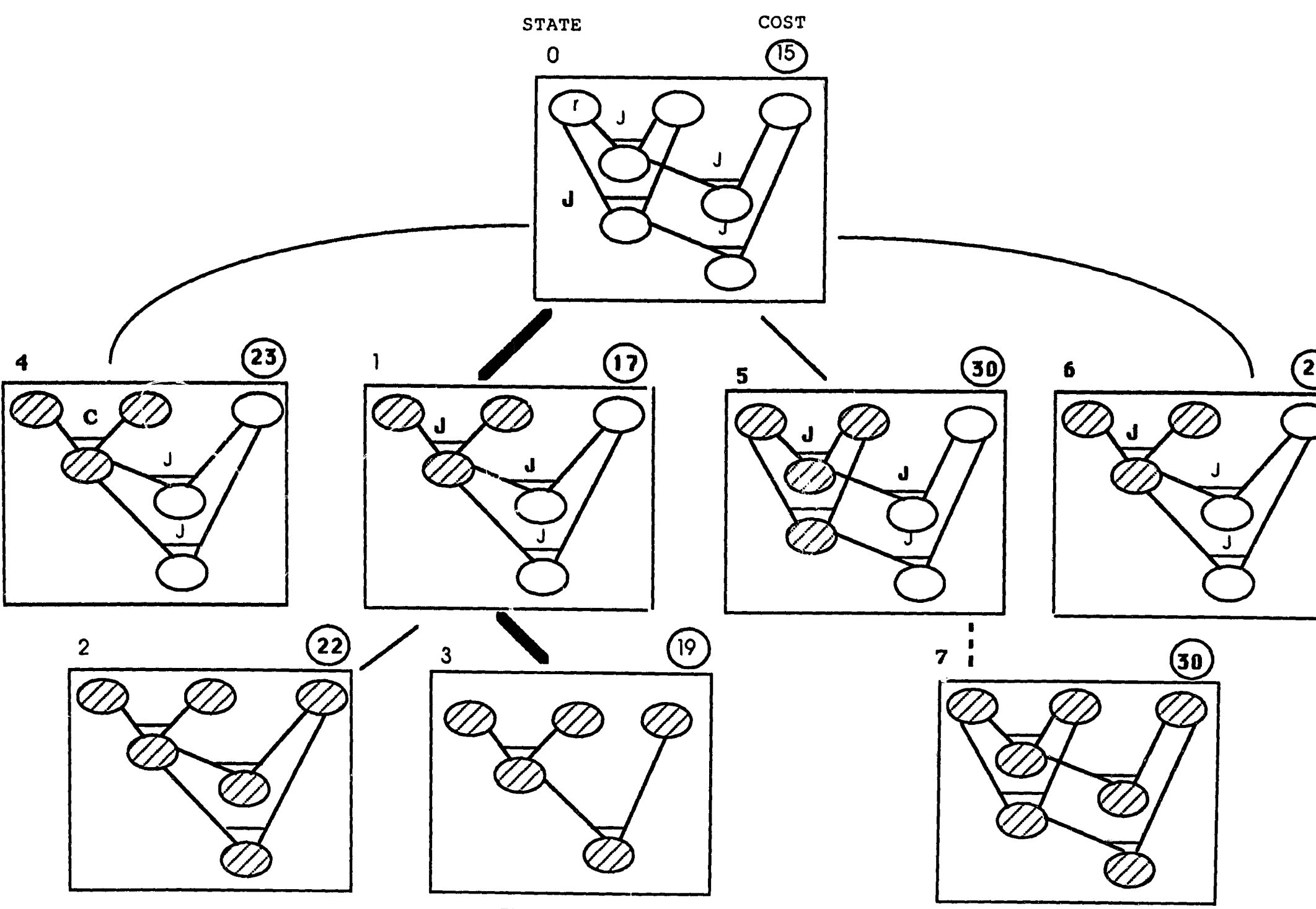

Fig. 14. The search tree generated by MTP. 


\section{Discussion}

Suppose that we have two queries $Q T_{2}$ and $Q T_{3}$ where $Q T_{2}$ is a superquery of $Q T_{3}$ as shown in Fig. 13. The total processing costs $T_{-} \operatorname{COS} T_{I}$, using individual query processing strategies, and $T_{-} C O S T_{M}$, using $M T P$, are

$$
\begin{gathered}
T_{-} C O S T_{I}=\text { cost of executing } Q T_{2}+\text { cost of executing } Q T_{3} \\
T_{-} C O S T_{M}=\text { cost of executing } Q T_{2}+\psi^{\prime}\left(\text { uop, } \operatorname{VIEW}\left(Q T_{2}\right)\right)+\Delta,
\end{gathered}
$$

where $\psi^{l}\left(\right.$ uop, $\left.\operatorname{VIEW}\left(Q T_{2}\right)\right)$ is the local processing cost to obtain $\operatorname{VIEW}\left(Q T_{3}\right)$ from the result of $Q T_{2}$, and where $\Delta$ is the overhead of executing $M T P$. The main factor in $\Delta$ is $C P U$ time associated with running $M T P$. The $I / O$ cost is assumed to be small since the size of the knowledge base containing the semantic knowledge is unlikely to be large in our problem. On the other hand, the cost of executing $Q T_{3}$ usually involves intersite data communication costs in addition to $C P U$ and $I / O$ costs. Thus one can conjecture that using a multiple query processing strategy such as $M T P$ will be beneficial in environments where communication costs are significant and where the queries and ICs are such that some commonality can be found between the given access plans. This conjecture is strengthened by the promising empirical results recently reported in [33], where multiple query optimization produced a decrease of $20-50 \%$ in both $C P U$ and $I / O$ time in a series of experiments on a centralized database, and by the seminal work of King [15] on semantic query optimization.

The central issue for the cost of running MTP lies in the performance of the heuristic search. First, we claim that the number of feasible states in the search space is $O\left(s \cdot k^{k} \cdot m^{l}\right)$, where $s$ is the number of sites, $k$ the number of queries, $l$ the length of common access plans, and $m$ is the average number of superqueries which subsume a subset $Q T$ of the set of $k$ queries but do not subsume any superset of $Q T$. This result is proved in [25]. In brief, assums that there is at least one superquery which subsumes any subset of the $k$ queries (worst case analysis). The question is then how many collective query execution plans can be generated. This problem is equivalent to the problem of partitioning a set of $k$ elements where each collective query execution plan corresponds to a partition. It is known that the total number of partitions of a set with cardinality $k$ is $B_{k}$ where $B_{k}$ is the $k$ th Bell number,

$$
B_{k}=\sum_{i=0}^{k-1}\left(\begin{array}{c}
k-1 \\
i
\end{array}\right) B_{i}
$$

where $B_{0}=1 . B_{k}$ is almost of order $k^{k}$ for large $k$; for $k=15$, it is almost $10^{9}$. Therefore, for $k$ queries, the number of partitions on the set $Q T$ of queries $\cong O\left(k^{k}\right)$. For each partition, there are $m^{l}$ possible state transitions. Thus, the total number of state transitions $\cong$ $O\left(k^{k} \cdot m^{l}\right)$. If in addition joins can be performed at any site, i.e. if we relax the first condition in the assumption of fixed access order, then the total number of state transitions is $O\left(s \cdot k^{k} \cdot m^{l}\right)$.

A straightforward enumeration of all the possible collective execution plans, without using any inference-guiding heuristics, is thus computationally intractable either for the case where the number of queries is not small, or for a real-time environment in which the overhead due to enforcing the multiple query processing strategy cannot be overlooked.

On the other hand, it is difficult to formally evaluate the performance of the heuristic search of $M T P$, especially due to its dependence on the knowledge base and thus on the predicate conditions of individual queries. As argued in [27], a worst case analysis assumes 
that $A^{*}$ exhibits its poorest performance; the average performance of $A^{*}$ is often significantly better. To quantify this statement, one needs to develop a probabilistic model of MTP and to apply the results in [27], Chap. 6. This is beyond the scope of this paper. However, we point out that since there are only a few types of rules which can activate state transitions, the search should be efficient if proper semantic knowledge is available.

\section{Conclusion}

We have introduced a new multiple query processing strategy that makes use of functional dependencies and semantic integrity constraints to determine subset relationships between intermediate results of different queries. The concept of the conventional query graph is extended to represent distributed query processing strategies by including site information. Given some semantic knowledge, the least cost solution is found by a rule-based expert system, Multiple Transaction Processor (MTP), in which the planning technique is combined with a search method. We define the cost function $(g)$ and the estimated cost function $(\hat{h})$ for the case of distributed query processing. We also provide a proof that the estimated function $(\hat{h})$ is always underestimating to ensure that an optimal solution will be produced by the $A^{*}$ algorithm.

\section{Acknowledgement}

The authors gratefully acknowledge the critical analysis and suggestions by the referees.

\section{References}

[1] P.A. Bernstein, N. Goodman, E. Wong, C.L. Reeve and J.B. Rothnie, Query processing in a system for distributed databases (SDD-1), ACM Trans. on Database Systems, vol. 6, no. 4 (Dec. 1981) 602-625.

[2] R. Blankinship, D. Dorris and A.R. Hevner, The file allocation problem - A survey and annotated bibliography, MS/S 85-013, Database Systems Research Center, University of Maryland (June 1985).

[3] S. Ceri, and G. Pelagatti, Distributed Databases, Principles and Systems (McGraw-Hill, 1984).

[4] U.S. Chakravarthy, D.H. Fishman and J. Minker, Semantic query optimization in expert systems and database systems, in Proc. 1st Intl. Workshop on Expert Data Base Systems, L. Kerschberg (Ed.) (Benjamin/Cummings Publ. Co. Inc.) 659-674 (1986).

[5] U.S. Chakravarthy and J. Minker, Processing multiple queries in database system, IEEE Database Eng., vol. 5 (Sept. 1982) 38-43.

[6] S. Finkelstein, Common expression analysis in database applications, in Proc. 1982 ACM-SIGMOD Int. Conf. Management of Data, 235-245.

[7] M.S. Fox and J. McDermott, The role of databases in knowledge-based systems, CMU-RI-TR-86-3, Department of Computer Science, Carnegie-Mellon University (Feb. 1986).

[8] J. Grant and J. Minker, Optimization in deductive and conventional relational database systems, in Advances in Data Base Theory, Vol. 1, H. Gallaire, J. Mirker and J.-M. Nicolas (Eds) (Plenum, New York, 1981) 195-234.

[9] A. Guttman, New Features for Relational Database Systems to Support CAD Applications, Ph. D Thesis, University of California, Berkeley (June 1984).

[10] P.A.V. Hall, Optimization of single expressions in a relational database system, IBM J. Res. Develop., vol. 20 (May 1976) 144-257.

[11] M.M. Hammer and S.B. Zdonik, Jr., Knowledge-based query processing, in Proc. Sixth Intl. Conf. on VLDB, Montreal (Oct. 1980) 137-147.

[12] M. Jarke, J. Clifford and Y. Vassiliou, An optimizing PROLOG front-end to a relational query system, in Proc. 1984 ACM-SIGMOD Intl. Conf. Management of Data, Boston, MA (June 1984). 
[13] M. Jarke and J. Koch, Query optimization in database systems, Computing Surveys, vol. 16, no. 2 (June 1984) 111-152.

[14] M. Jarke, Common subexpression isclation in multiple query optimization, in Query Processing in Database Systems, W. Kim, D. Reiner, and D. Batory (Eds.) (Springer-Verlag, 1985) 191-205.

[15] J.J. King, Query Optimization by Semantic Reasoning (UMI Research Press, Ann Arbor, Michigan, 1981).

[16] W. Kim, Global optimization of relational queries: A first step, Query Processing in Database Systems, W. Kim, D. Reiner and D. Batory (Eds.) (Springer-Verlag, 1985).

[17] S. Lafortune and E. Wong, A state transition model for distributed query processing, ACM Trans. on Database Systems, vol. 11, no. 3 (Sept. 1986) 294-322.

[18] R.K. Lindsay, B.G. Buchanan, B.A. Feigenbaum and J. Lederberg, Applications of Artificial Intelligence for Organic Chemistry: The Dendral Project (McGraw-Hill, 1980).

[19] B.G. Lindsay, L.M. Hass, C. Mohan, P.F. Wilms et al., Computation and communication in $R^{*}$ : a distributed database manager, ACM Trans. on Computer Systems, vol. 2, no. 1 (Feb. 1984) 24-38.

[20] L. Lilien and B. Bhargava, Database integrity block construct, IEEE Trans. on Software Eng., vol. SE-11, no. 9 (Sept. 1985) 865-885.

[21] G. Lohman, C. Mohan, L. Hass, D. Daniels, B. Lindsay, P. Selinger and P. Wilms, Query processing in $R^{*}$, in Query Processing in Database Systems, W. Kim, D. Reiner and D. Batory (Eds.) (Springer-Verlag, 1985).

[22] D. Maier, The Theory of Relational Databases (Computer Science Press, Rockville, Maryland, 1983).

[23] J. McDermott, R1: A rule-based configurer of computer systems, Artificial Intelligence, vol. 19 (1982) 39-88.

[24] N.J. Nilsson, Principles of Artificial Intelligence (Tioga Publishing Co., CA, 1980).

[25] J.T. Park, A Knowledge-Based Approach to Míultiple Transaction Processing and Distributed Database Design, Ph.D. Thesis, University of Michigan, Ann Arbor (1987).

[26] J.T. Park and T.J. Teorey, A knowledge-based approach to multiple query processing in distributed database systems, Proc. 1987 ACM-IEEE Fall Joint Computer Conference, Dallas, TX, (Oct. 1987) 461-468.

[27] J. Pearl, Heuristics: Intelligent Search Strategies for Computer Problem Solving (Addison-Wesley, 1984).

[28] N. Roussopoulos, View indexing in relational databases, ACM Trans. on Database Systems, vol. 7, no. 2 (June 1982) 258-290.

[29] N. Roussopoulos, The logical access path schema of a database, IEEE Trans. on Software Eng., vol. SE-8, no. 6 (Nov. 1982) 563-573.

[30] E.D. Sacerdoti, A structure for plans and behavior (American Elsevier, New York, 1977).

[31] M. Schkolnick, A clustering algorithm for hierarchical structures, ACM Trans. on Database Systems, vol. 2. no. 1, pp. 27-44 (Mar. 1977).

[32] T.K. Sellis, Global query optimization, in Proc. 1986 ACM-SIGMOD Intl. Conf. Management of Data, Washington, D.C. (May 1986) 191-205.

[33] T.K. Scllis, Multiple-Query Optimization, ACM Trans. on Database Systems, Vol. 13, No. 1 (March 1988) 23-52.

[34] B. Shneiderman and V. Goodman, Batched searching of sequential and tree structured files, ACM Trans. on Database Systems, vol. 1, no. 3 (Sept. 1976) 268-275.

[35] S.T. Shenoy and Z.M. Ozsoyoglu, A system for semantic query optimization, in Proc. 1987 ACM-SIGMOD Int. Conf. Management of Data. 181-195.

[36] S.Y.W. Su, K.P. Mikkilineni, R.A. Liuzzi and Y.C. Chow, A distributed query processing strategy using decomposition, pipelining and intermediate result sharing techniques, in Proc. IEEE 1986 Intl. Conf. on Data Engineering, Los Angeles, CA, (Feb. 1986) 94-102.

[37] T.J. Teorey and J.P. Fry, Design of Database Structures (Prentice-Hall, Englewood Cliffs, N.J., 1982).

[38] P. Valduriez, Join indices, ACM Trans. on Database Systems, vol. 12, no. 2, (June 1987) 218-246.

[39] C.T. Yu and C.C. Chang. Distributed query processing, Computing Surveys, vol. 16, no. 4 (Dec. 1984) 399-433. 